\title{
Cloud Structure of Three Galactic Infrared Dark Star-forming Regions from Combining Ground- and Space-based Bolometric Observations
}

\author{
Yuxin $\operatorname{Lin}^{1,2}$, Hauyu Baobab Liu ${ }^{3}$, James E. Dale ${ }^{4}$, Di Li ${ }^{1,5}$, Gemma Busquet ${ }^{6}$, Zhi-Yu Zhang ${ }^{3,7}$, \\ Adam Ginsburg $^{3}$, Roberto Galván-Madrid ${ }^{8}$, Attila Kovács ${ }^{9}$, Eric Koch ${ }^{10}$, Lei Qian ${ }^{1,5}$, Ke Wang ${ }^{3}$, \\ Steve Longmore ${ }^{11}$, Huei-Ru Chen ${ }^{12}$, and Daniel Walker ${ }^{11}$ \\ ${ }^{1}$ National Astronomical Observatories, Chinese Academy of Sciences, China \\ ${ }^{2}$ Max-Planck-Institut für Radioastronomie, D-53121 Bonn, Germany; ylin@mpifr-bonn.mpg.de \\ ${ }^{3}$ European Southern Observatory (ESO), Karl-Schwarzschild-Str. 2, D-85748 Garching, Germany \\ ${ }^{4}$ Centre for Astrophysics Research, University of Hertfordshire, College Lane, Hatfield, AL10 9AB, UK \\ ${ }^{5}$ Key Laboratory of Radio Astronomy, Chinese Academy of Sciences, China \\ ${ }^{6}$ Institut de Ciències de l'Espai (IEEC-CSIC), Campus UAB, Carrer de Can Magrans S/N, E-08193, Barcelona, Catalunya, Spain \\ ${ }^{7}$ Institute for Astronomy, University of Edinburgh, Royal Observatory, Blackford Hill, Edinburgh EH9 3HJ, UK \\ ${ }^{8}$ Instituto de Radioastronomía y Astrofísica, UNAM, Apdo. Postal 3-72 (Xangari), 58089 Morelia, Michoacán, México \\ ${ }^{9}$ California Institute of Technology 301-17, 1200 East California Boulevard, Pasadena, CA 91125, USA \\ ${ }^{10}$ Department of Physics, University of Alberta, 4-181 CCIS, Edmonton, AB T6G 2E1, Canada \\ ${ }^{11}$ Astrophysics Research Institute, Liverpool John Moores University, 146 Brownlow Hill, Liverpool L3 5RF, UK \\ ${ }^{12}$ Institute of Astronomy and Department of Physics, National Tsing Hua University, Hsinchu, Taiwan \\ Received 2016 October 29; revised 2017 April 6; accepted 2017 April 7; published 2017 May 2
}

\begin{abstract}
We have modified the iterative procedure introduced by Lin et al., to systematically combine the submillimeter images taken from ground-based (e.g., CSO, JCMT, APEX) and space (e.g., Herschel, Planck) telescopes. We applied the updated procedure to observations of three well-studied Infrared Dark Clouds (IRDCs): G11.11-0.12, G14.225-0.506, and G28.34+0.06, and then performed single-component, modified blackbody fits to each pixel to derive $\sim 10^{\prime \prime}$ resolution dust temperature and column density maps. The derived column density maps show that these three IRDCs exhibit complex filamentary structures embedded with rich clumps/cores. We compared the column density probability distribution functions (N-PDFs) and two-point correlation (2PT) functions of the column density field between these IRDCs with several OB-cluster-forming regions. Based on the observed correlation between the luminosity-to-mass ratio and the power-law index of the N-PDF, and complementary hydrodynamical simulations for a $10^{4} M_{\odot}$ molecular cloud, we hypothesize that cloud evolution can be better characterized by the evolution of the (column) density distribution function and the relative power of dense structures as a function of spatial scales, rather than merely based on the presence of star-forming activity. An important component of our approach is to provide a model-independent quantification of cloud evolution. Based on the small analyzed sample, we propose four evolutionary stages, namely, cloud integration, stellar assembly, cloud pre-dispersal, and dispersed cloud. The initial cloud integration stage and the final dispersed cloud stage may be distinguished from the two intermediate stages by a steeper than -4 power-law index of the N-PDF. The cloud integration stage and the subsequent stellar assembly stage are further distinguished from each other by the larger luminosity-to-mass ratio $\left(>40 L_{\odot} / M_{\odot}\right)$ of the latter. A future large survey of molecular clouds with high angular resolution may establish more precise evolutionary tracks in the parameter space of N-PDF, 2PT function, and luminosity-to-mass ratio.
\end{abstract}

Key words: ISM: structure - stars: formation - submillimeter: ISM

\section{Introduction}

Infrared dark clouds (IRDCs) are dense, cold molecular gas clouds that efficiently absorb the Galactic bright mid-infrared emission (Hennebelle et al. 2001; Simon et al. 2006; Peretto \& Fuller 2009). Due to the high molecular gas mass and low bolometric luminosity of these objects, they are considered candidate progenitors of massive stars or star clusters (Carey et al. 1998; Sridharan et al. 2005; Ragan et al. 2006). The density distribution in the IRDCs may reflect the dominant physical mechanisms during the formation of these clouds and can be used to gauge their subsequent gravitational contraction. In particular, the widely resolved filamentary morphologies of the IRDCs indicate that they will likely undergo a hierarchical collapse (Inutsuka \& Miyama 1997; Vázquez-Semadeni et al. 2007, 2009). The details of how IRDCs collapse and eventually evolve into luminous OB-cluster-forming molecular clouds remain uncertain. Not all IRDCs will evolve to form massive stars (Peretto \& Fuller 2009; Kauffmann \& Pillai 2010). How to distinguish those possible progenitors of high-mass stars or clusters from a large sample of IRDCs quantitatively and qualitatively remains not elaborately resolved.

To pilot the systematic quantification for the similarity and the difference between the IRDCs and the more evolved OB-clusterforming regions, we have performed high angular resolution mapping observations of the $350 \mu \mathrm{m}$ dust emission toward three very well studied IRDCs, namely, G11.11-0.12, G14.225 -0.506 , and G28.34+0.06, using the Caltech Submillimeter Observatory (CSO) Submillimetre High Angular Resolution Camera II (SHARC2). We also performed deep $870 \mu \mathrm{m}$ mapping observations on G14.225-0.506, using the Large Apex BOlometer CAmera (APEX-LABOCA; Siringo et al. 2009). Table 1 summarizes the basic properties of the selected target sources. References for these observed IRDCs can be found: for G11.11-0.12 in Pillai et al. (2006), Henning et al. (2010), 
Table 1

Source Information

\begin{tabular}{|c|c|c|c|c|c|}
\hline Target Source & $\begin{array}{l}\text { R.A. } \\
\text { (J2000) }\end{array}$ & $\begin{array}{l}\text { Decl. } \\
(\mathrm{J} 2000)\end{array}$ & $\begin{array}{l}\text { Distance }^{\mathrm{a}} \\
(\mathrm{kpc})\end{array}$ & $\begin{array}{l}\text { Mass }^{\mathrm{b}} \\
\left(M_{\odot}\right)\end{array}$ & $\begin{array}{c}\text { Luminosity }^{\mathrm{b}} \\
\left(L_{\odot}\right)\end{array}$ \\
\hline G11.11-0.12 & $19^{\mathrm{h}} 10^{\mathrm{m}} 13^{\mathrm{s}} .00$ & $09^{\circ} 06^{\prime} 00^{\prime \prime} 0$ & $3.6 \pm 0.72$ & $9.3 \times 10^{4}$ & $6.0 \times 10^{5}$ \\
\hline G14.225-0.506 & $18^{\mathrm{h}} 47^{\mathrm{m}} 36^{\mathrm{s}} \cdot 43$ & $-01^{\circ} 59^{\prime} 02^{\prime \prime} 5$ & $1.98_{-0.12}^{+0.13 c}$ & $1.9 \times 10^{4}$ & $1.95 \times 10^{5}$ \\
\hline
\end{tabular}

Notes.

${ }^{\text {a }}$ We tentatively quote a $20 \%$ distance uncertainty for kinematic distances.

$\mathrm{b}$ Total masses were summed from our derived column density maps (Figures 1-3) above each source's measured column density threshold. The total bolometric luminosity is calculated by integrating from $0.1 \mu \mathrm{m}$ to $1 \mathrm{~cm}$ of the obtained SED for each pixel of column density above threshold, and adding all the values in each field. For a detailed procedure of how these quantities are calculated, we refer to Lin et al. (2016).

${ }^{\mathrm{c}}$ Distance measurement by parallaxes of methanol masers (Xu et al. 2011; Wu et al. 2014).

Wang et al. (2014), Wang (2015), and Pillai et al. (2015); for G28.34+0.06 in Wang et al. (2008, 2011, 2012), Wang (2015), Zhang et al. (2009, 2015), Chen et al. (2011), and Butler et al. (2014); and for G14.225-0506 in Busquet et al. (2013, 2016) and Santos et al. (2016).

To obtain a finest possible angular resolution, and to precisely constrain physical properties on all angular scales, we build on procedures suggested by Liu et al. (2015) and Lin et al. (2016) to combine the $350 \mu \mathrm{m}$ images and other groundbased (JCMT-SCUBA2 and APEX-LABOCA, see Section 2) $850 / 870 \mu \mathrm{m}$ images with space telescope observations, and then derive the dust temperature and dust/gas column density maps with $\sim 10^{\prime \prime}$ angular resolution. In addition, we derived the statistical measures of the cloud structures for these IRDCs, and then compare with the same analysis for seven very luminous $\left(L_{\text {bol }}>10^{6} L_{\odot}\right)$ OB-cluster-forming molecular clouds published in Lin et al. (2016). Finally, we performed numerical hydrodynamics simulations to demonstrate how the cloud morphology and the derived statistical quantities evolve with time. The observations and data analysis procedures are outlined in Section 2. Results are provided in Section 3. A comparison among the observed star-forming regions and the comparison with numerical hydrodynamics simulations, are given in Section 4. The main conclusion and ending remarks are listed in Section 5.

\section{Observations}

We introduce our CSO SHARC2 $350 \mu \mathrm{m}$ observations and data reduction in Section 2.1. The APEX-LABOCA observations and data calibrations are introduced in Section 2.2. Section 2.3 outlines the archival data we included for the spectral energy distribution (SED) analysis. The procedures for producing the final images and SED fitting are given in Section 2.4 .

\subsection{CSO-SHARC2 Observations}

High angular resolution, ground-based continuum observations at $350 \mu \mathrm{m}$ toward the three IRDCs G11.11-0.12, G28.34 +0.06 , and G14.225-0.506 were carried out using the SHARC2 bolometer array, installed on the CSO Telescope (PI: H. B. Liu). The array consists of $12 \times 32$ pixels. ${ }^{13}$ The simultaneous field of view (FOV) provided by this array is 2 .59 $\times 0$ '.97, and the diffraction limited beam size is $\sim 8$.' 8 . The data of G11.11-0.12 were acquired on 2014 March 28

\footnotetext{
13 Approximately $85 \%$ of these pixels work well according to the online documentation: http://www.astro.caltech.edu/ sharc/.
}

$\left(\tau_{225 \mathrm{GHz}} \sim 0.06\right)$, with an on-source exposure time of 90 minutes. G14.225-0.506 and G28.34+0.06 were observed on 2014 March $27\left(\tau_{225} \mathrm{GHz} \sim 0.05\right)$, with 80 and 50 minutes of on-source exposure time, respectively. The telescope pointing and focusing were checked every $1.5-2.5 \mathrm{hr}$. Mars was observed for absolute flux calibration. We used the standard $10^{\prime} \times 10^{\prime}$ on-the-fly box scanning pattern, and the scanning center for each source is listed in Table 1. Basic data calibration was carried out using the CRUSH software package (Kovács 2008). We used the -faint option of the CRUSH software package during data reduction, which optimized the reconstruction of the faint and compact sources with the cost of the more aggressive filtering of extended emission. Nevertheless, the extended emission components will ultimately be complemented by the observations of space telescopes (see Section 2.4 for more details). The final calibrated map was smoothed with a Gaussian kernel with $2 / 3$ beam FWHM (-faint option) to an angular resolution of 9 ". 6 for an optimized sensitivity and source reconstruction, and the rms noise levels we measured from the approximately emission-free areas of 56, 53, and $60 \mathrm{mJy}^{\text {beam }}{ }^{-1}$ for $\mathrm{G} 11.11-0.12, \mathrm{G} 28.34$ +0.06 , and G14.225-0.506, respectively.

\subsection{APEX-LABOCA Observations}

The $870 \mu \mathrm{m}$ submillimeter continuum observations toward G14.225-0.506 were conducted (PI: G. Busquet) using the LABOCA bolometer array, installed on the Atacama Pathfinder EXperiment (APEX). ${ }^{14}$ The array consists of 259 channels arranged in nine concentric hexagons around the central channel. The FOV of the array is $11 ! 4$, and the angular resolution of each beam is 18 . $^{\prime \prime} \pm 1^{\prime \prime}$. The observations were carried out on 2008 August 24 and 31 under weather conditions with zenith opacity values that ranged from 0.15 to 0.24 at $870 \mu \mathrm{m}$. The observations were performed using a spiral raster mode mapping, providing a fully sampled and homogeneously covered map in an area of $15^{\prime} \times 15^{\prime}$.

Calibration was done using observations of Mars as well as secondary calibrators. The absolute flux uncertainty is estimated to be $\sim 8 \%$. Pointing was checked every hour, finding an rms pointing accuracy of $2^{\prime \prime}$, and focus settings were performed once per night and during the sunset. The data was reduced using the MiniCRUSH software package (see Kóvacs 2008). The data reduction process included flat-fielding,

\footnotetext{
14 This publication is based on data acquired with the Atacama Pathfinder Experiment (APEX). APEX is a collaboration between the Max-Planck-Institut fur Radioastronomie, the European Southern Observatory, and the Onsala Space Observatory.
} 
opacity correction, calibration, correlated noise removal, and de-spiking. Further details of the data acquisition and data reduction are described in Busquet et al. (2016).

\subsection{Archival Herschel, Planck, and James Clerk Maxwell Telescope (JCMT) Data}

We retrieved the available night observations ${ }^{15}$ of JCMT Submillimetre Common-user Bolometer Array 2 (SCUBA2; Chapin et al. 2013; Dempsey et al. 2013; Holland et al. 2013) at $850 \mu \mathrm{m}$ from the online data archive. (Program ID is M11BEC30 for G11.11-0.12 and G28.34+0.06.) Ancillary data also includes level 2.5 and level 3 processed, archival Herschel ${ }^{16}$ images, which were taken by the Herschel Infrared Galactic Plane (Hi-GAL) survey (Molinari et al. 2010) at 70/160 $\mu \mathrm{m}$ using the PACS instrument (Poglitsch et al. 2010) and at 250/350/500 $\mu \mathrm{m}$ using the SPIRE instrument (Griffin et al. 2010). Observation IDs for G14.225-0.506 are 1342218997 and 1342219000, for G11.11-0.12 they are 1342204952 and 1342218965 , and for G28.34+0.06 it is 1342218694. Planck/High Frequency Instrument (HFI) $353 \mathrm{GHz}$ images are also used. For our combination purpose, we use the Herschel SPIRE extended emission products from the data base. The Planck $353 \mathrm{GHz}$ images, which are in units of $\mathrm{K}_{\mathrm{CMB}}$, are converted to Jy beam ${ }^{-1}$ (Planck HFI Core Team et al. 2011a, 2011b; Zacchei et al. 2011).

\subsection{Image Combination and Derivations of Dust Column Density and Temperature}

Our procedure to combine images taken from ground-based and space telescope observations in the Fourier domain, and then iteratively derive the high angular resolution dust temperature and dust/gas column density images, is similar to what was introduced in Lin et al. (2016). Since the observed IRDCs for the present paper have relatively low temperatures, it is particularly important to precisely determine the long wavelength part of the spectrum. Therefore, we additionally included the following steps to improve the quality of the combined $850 \mu \mathrm{m}$ image before making our final modified blackbody SED fits.

We extrapolated the $850 \mu \mathrm{m}$ flux from SED fits to Herschel PACS $160 \mu \mathrm{m}$ and SPIRE $250 / 350 / 500 \mu \mathrm{m}$. In these fits, we fixed the dust emissivity index $\beta$ to 1.8 , which is the mean value measured for the Galactic disk (Planck Collaboration et al. 2011, 2014). The Herschel-extrapolated $850 \mu \mathrm{m}$ image has the same resolution as SPIRE $500 \mu \mathrm{m}, \sim 37^{\prime \prime}$. Then we proceeded to combine the above mentioned $850 \mu \mathrm{m}$ image with Planck $353 \mathrm{GHz}$, and then further combined the result with the SCUBA2 $850 \mu \mathrm{m}$ image. In this way, the $>5^{\prime}$ scale and the $<3^{\prime}-4^{\prime}$ scale structures are dominantly constrained by the Planck and the SCUBA2 images, respectively. The role of the Herschel-extrapolated $850 \mu \mathrm{m}$ image is to complement the small range of spatial scales, which is poorly sampled by Planck and SCUBA2.

\footnotetext{
15 http://www.eaobservatory.org/jcmt/science/archive/guide/

${ }^{16}$ Herschel is an ESA space observatory with science instruments provided by European-led Principal Investigator consortia and with important participation from NASA.
}

\section{Results}

\subsection{Molecular Cloud Structures and Temperature Distribution}

Figures 1-3 show the derived dust temperature and gas column density maps, assuming a gas to dust mass ratio of 100 . For detailed calculations and procedure, see Appendix A and Lin et al. (2016). The derived gas masses of these three IRDCs range in $\sim 3-9 \times 10^{4} M_{\odot}$. The overall gas masses and bolometric luminosities derived from these maps are summarized in Table 1 .

The column density maps of these IRDCs present considerably different overall morphologies. On the $\sim 30 \mathrm{pc}$ scale, IRDC G11.11-0.12 appears as an integral shaped filament, which shows some wiggles on $\sim 10 \mathrm{pc}$ scales. In addition, the high angular resolution we achieve reveals that the large-scale filament may in fact consist of bundles of filaments. The majority of dense gas structures in G11.11-0.12 have dust temperature lower than $20 \mathrm{~K}$. We are able to identify one internally heated source embedded approximately at the center of the overall filament, where previous observations have found water masers, Class II methanol masers, and compact centimeter continuum sources (Pillai et al. 2006; Rosero et al. 2014; Wang et al. 2014). There are several isolated heating sources, which are projected adjacent or well outside of the dense filament. How many of those heating sources are directly associated with this molecular cloud is not yet certain.

IRDC G14.225-0.506 is resolved into a web of dense gas filaments, which seem to align in two preferred directions (see also the discussion in Busquet et al. 2013). The two most significant column density peaks in Figure 2 correspond to the two previously known relatively massive $\left(\sim 10^{3} M_{\odot}\right), \sim 0.5 \mathrm{pc}$ scale molecular clumps. ${ }^{17}$ The northern massive molecular clump, namely Hub-N, is adjacent to a compact H II region, which presents a high dust temperature over $1^{\prime}-2^{\prime}$ angular scales. The rest of the dense structures have $\sim 18-20 \mathrm{~K}$ dust temperature in general. There are several heated sources embedded in the filamentary structures in G14.225-0.506.

The dominant dense gas structures in G28.34+0.06 can be described by a massive molecular clump in the north (P2, see Carey et al. 2000; Wang et al. 2008; Zhang et al. 2009), which is connected with a dense gas filament in the south. The southern gas filament has several embedded clumps.

Star-formation in the P2 clump is already active. The P2 clump is internally heated by the embedded young stars, and shows higher than $25 \mathrm{~K}$ dust temperature. Dust temperature in the southern molecular clumps remains under $20 \mathrm{~K}$.

\subsection{Column Density Probability Distribution Function, Column Density Complementary Cumulative Distribution Function, and Two-point Correlation Function}

To systematically quantify the dense gas distributions in the observed IRDCs, and thereby permit comparisons with other observations and theoretical models, we perform analyses of

\footnotetext{
17 We follow the existing nomenclature in the literature (e.g., Zhang et al. 2009; Wang et al. 2011; Liu et al. 2012a, 2012b). In this way, massive molecular clumps refer to structures with sizes of $\sim 0.5-1 \mathrm{pc}$, massive molecular cores refer to the $<0.1 \mathrm{pc}$ size structures embedded within a clump, and condensations refer to the distinct molecular substructures within a core. Fragmentation refers to the dynamical process that produces or enhances multiplicity. Molecular filaments refer to the geometrically elongated molecular structures, and molecular arms refer to segments of molecular filaments that are located within the $\lesssim 1 \mathrm{pc}$ radii of molecular clumps and may not be fully embedded within molecular clumps.
} 


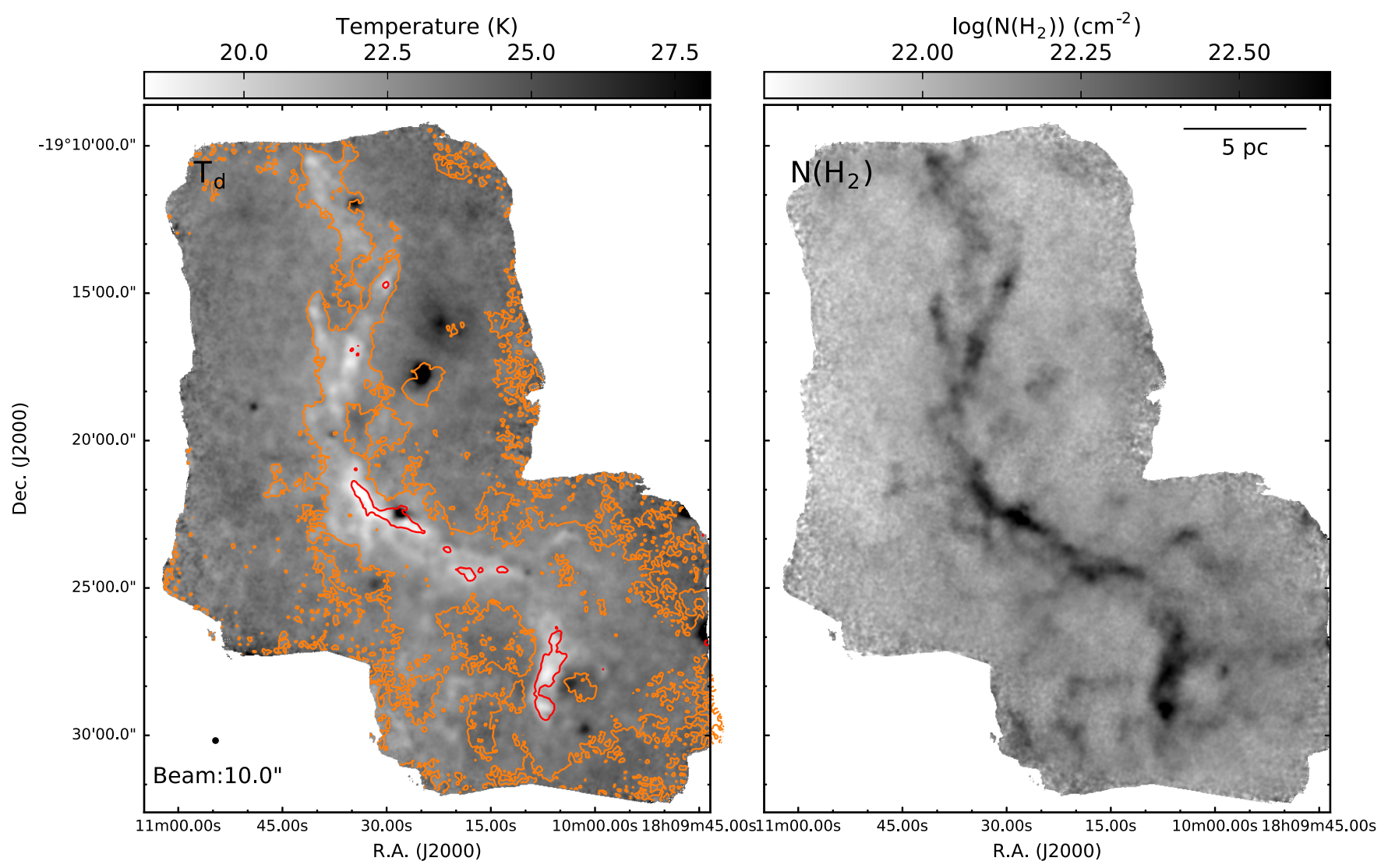

Figure 1. Dust temperature and column density maps of G11.11-0.12, derived based on fitting modified blackbody spectra iteratively to the Herschel-PACS $70 / 160 \mu$ m, Herschel-SPIRE $250 \mu \mathrm{m}$, and the combined $350 / 850 \mu \mathrm{m}$ images. The detailed procedures can be found in Section 2.4 and Appendix A. Two contours are indicated in the temperature map of column density levels $\left.\log _{10}\left(\mathrm{~N}_{(\mathrm{H}}\right)\right)$ of 22.14 and 22.47. See also Figure 4 for an explanation of how these contours probe the overall column density distribution.

the column density probability distribution functions (N-PDF), column density complementary cumulative distribution function (N-CCDF), and the two-point correlation functions (2PT) of gas column density.

The N-PDFs of the three IRDCs are presented in Figure 4. For the sake of enabling quantitative comparisons with other observations, we approximate the components in the N-PDFs that have a decreasing slope at lower column densities by a log-normal distribution function, and approximate the components that have a near constant slope at higher column densities by a power-law distribution function. In the later discussion, we will refer to these as the "log-normal" and "power-law" components. We emphasize that the functional forms we selected are merely approximations for the actual observed N-PDFs, which instead show richer features that cannot be described by a simple fit to these functional forms. Linking these functional forms to the underlying physics is not trivial, and one should not over-emphasize on the exact functional forms. We elaborate further on this point in Section 4.2 by examining comparisons of numerical hydrodynamic simulations.

We fit an overall power-law and a power-law starting from the optimal column density cutoff based on Maximum Likelihood Estimation (MLE; Clauset et al. 2009; Alstott et al. 2014) to the N-PDF of G28.34+0.06. The results of these two fits appear consistent. At the high column density end, however, the N-PDF of G28.34+0.06 shows an excess over the fitted power law. The N-PDFs of G14.225-0.506 and G11.11-0.12 are better described by a log-normal component in the lower column density part, in addition to the optimal power-law fit at the high column density end. Whether the log-normal component is included in the fit or not does not affect the results of the optimal power-law fit because of the MLE approach used to derive the optimal cutoff for power-law fit instead of jointly fitting the two functional forms.

The N-PDF of G14.225-0.506 presents an apparent deviation from the log-normal and power-law fits over a wide range of the normalized column density at $\eta \sim 1-3$, and therefore it is hard to define whether there is an excess or deficit at any range of $\eta$. In particular, a significant "bump" is seen around $\eta \sim 2.0-2.3$. The N-PDF of G11.11-0.12 decreases the most rapidly with increasing $\eta$. The fitted parameters are tabulated in Table 2 and are also labeled in each N-PDF panel in Figure 4.

The two-point correlation (2PT) function we used follows the same form as that in Kleiner \& Dickman (1984), but instead of calculating the correlation of column density fluctuations, we directly measure the correlation of column densities for each source across the observed field. The correlation strength at a separation scale (lag) of $l$ is calculated by

$$
S_{\mathrm{tr}}(l)=\frac{\langle X(\boldsymbol{r}) X(\boldsymbol{r}+1)\rangle_{l}}{\langle X(\boldsymbol{r}) X(\boldsymbol{r})\rangle_{l}},
$$



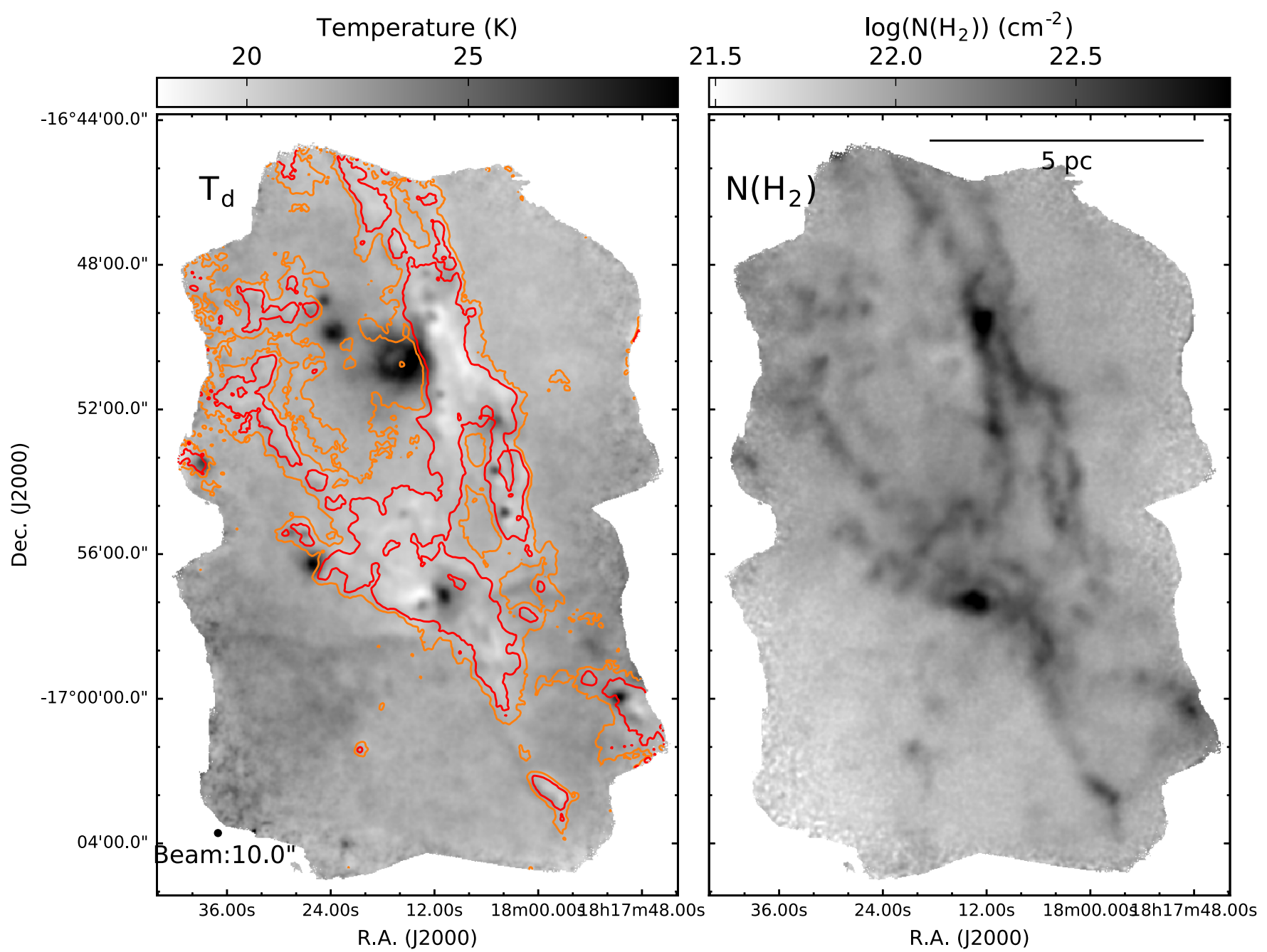

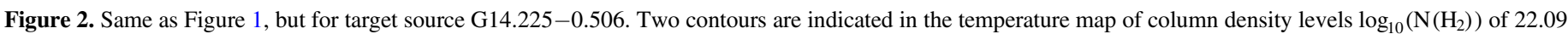
and 22.21 .

where $X(\boldsymbol{r})$ denotes the column density value at position $\boldsymbol{r}$, and the angle brackets are the average over all pairs of positions with a separation of $l$. The final form of the correlation function is normalized by the peak correlation strength to enable comparisons between different fields. For a more detailed description, we refer to Lin et al. (2016).

Figure 5 shows the 2PT functions of the three IRDCs overplotted with those of the active OB-cluster-forming molecular clouds taken from Lin et al. (2016). The 2PT functions of the three IRDCs, in general, show a smooth decay of correlation strengths over all spatial scales. This is in contrast with most of the active OB-cluster-forming regions, which rapidly decrease at small scales. The dominant elongated cylindrical filaments in G11.11-0.12 and G14.225-0.506 may naturally have flattened 2PT functions since, by definition, filaments have comparable column density distributions over a wide range of separations. For G28.34+0.06, we spatially resolved complex local over-densities scattered across the column density map. The multiple fluffy and scattered overdense substructures of this molecular cloud more closely resemble the cloud morphology of W43-south and G10.2-0.3, which also have similar 2PT functions (see Lin et al. 2016).

The homogeneity of the column density fields could be estimated by the range of plateaus in the $2 \mathrm{PT}$ functions. In light of this, G11.11-0.12 would present the least mass concentration since its 2PT function remains at an almost constant correlation strength of up to $\sim 6 \mathrm{pc}$. G14.225-0.506, which harbours two pronounced massive clumps, shows a steep decrease at lags of less than $1 \mathrm{pc}$, indicating a relatively significant concentrated distribution at small scales. The 2PT function of G28.34+0.06 falls between those of G11.11-0.12 and G14.225-0.506 with a slight decay at small scales $(<1 \mathrm{pc})$ and a constant correlation plateau to $\sim 4 \mathrm{pc}$. We note that the 2PT function plateaus on the few parsec scales may be related to the elongated nature of cloud structures.

Figure 6 presents the column density complementary cumulative distribution function (N-CCDF) of these molecular clouds (this is also defined as the dense gas mass function in some previous works; see details in Kainulainen \& Tan 2013; Ginsburg et al. 2015). We exclude W49A, in spite of its highest fraction of dense gas, to avoid a bias from its larger distance $(\sim 11 \mathrm{kpc})$. The dense gas fraction is closely associated with the star-formation rate, raised based on observations toward both galactic and extragalactic star-forming regions (Gao \& Solomon 2004; Wu et al. 2005; Lada et al. 2010). In Figure 6, we plot the N-CCDFs for all of the sources. The dashed gray reference lines indicate exponential decay of indexes between -0.06 to -0.12 . The N-CCDF of OB-cluster-forming 

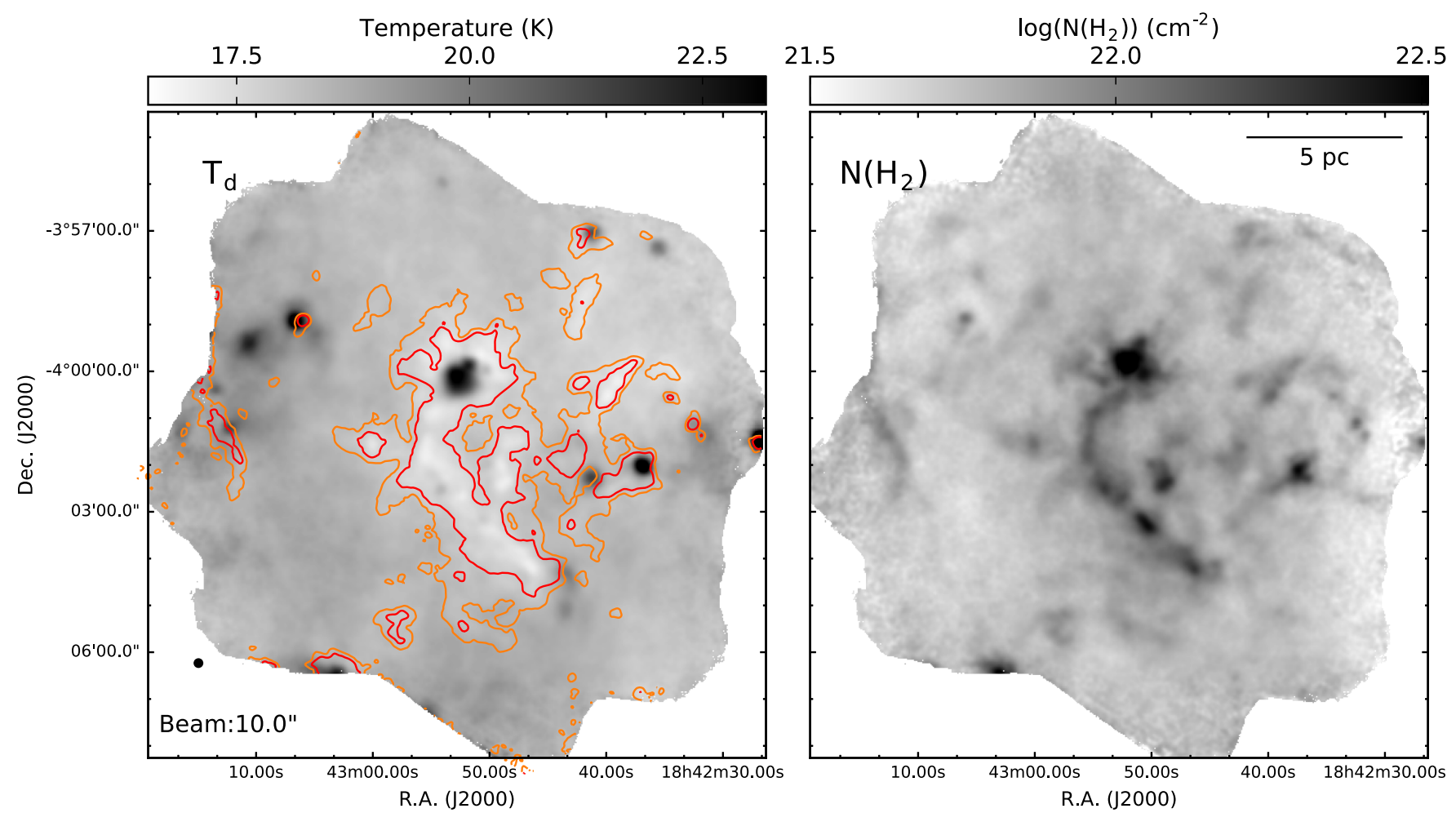

Figure 3. Same as Figure 1, but for target source G28.34+0.06. Two contours are indicated in the temperature map of column density levels log ${ }_{10}\left(\mathrm{~N}\left(\mathrm{H}_{2}\right)\right)$ of 21.83 and 21.91 .

Table 2

Fitting Results of Column Density Probability Distribution Functions (N-PDFs)

\begin{tabular}{|c|c|c|c|c|c|c|}
\hline Target Source & $\begin{array}{c}\text { Measured Threshold } \\
\log _{10}\left(\mathrm{~N}\left(\mathrm{H}_{2}\right)\right)\end{array}$ & $\begin{array}{l}\text { Mean Column Density } \\
\log _{10}\left(\mathrm{~N}\left(\mathrm{H}_{2}\right)\right)\end{array}$ & $\begin{array}{l}\text { Log-normal and Power-law Fits } \\
\qquad \sigma_{\eta}\end{array}$ & $\mu$ & $s_{0}$ & $s_{1}$ \\
\hline $\mathrm{G} 28.34+0.06$ & 21.70 & 21.73 & $\ldots$ & $\cdots$ & $-3.98(0.01)$ & $-3.86(0.02)$ \\
\hline G14.225-0.506 & 21.80 & 22.04 & 0.43 & 21.99 & $\ldots$ & $-4.12(0.01)$ \\
\hline G11.11-0.12 & 21.95 & 22.09 & 0.26 & 22.07 & $\ldots$ & $-6.75(0.09)$ \\
\hline
\end{tabular}

molecular clouds within our sample of IRDCs are generally more flattened in the high column density ends, except for G10.2-0.3 and G10.3-0.1. These sources hold a considerable mass fraction in the high column density regime. Despite its relatively high total mass, G11.11-0.12 has a sharp decrease of very dense gas and is consistent with its steep N-PDF powerlaw tail. We additionally plot two reference vertical lines representing a $10^{5} \mathrm{~cm}^{-2}, \sim 0.1 \mathrm{pc}$ core and a $10^{6} \mathrm{~cm}^{-2}$, $\sim 0.03 \mathrm{pc}$ core lying at the same distance.

The N-CCDF result we obtained for G11.11-0.12 is consistent with Herschel results obtained by Kainulainen et al. (2013b), where the authors also use near- and midinfrared absorption to derive an $\sim 2^{\prime \prime}$ column density map of this cloud. The large discrepancy of our column density map with their extinction derived one may mainly come from the resolution difference (and also the reprojection to a larger distance in our case). An $\sim 0.035 \mathrm{pc}$ resolution extinction map enables detections of embedded dense cores, which are expected to give a significant rise to N-CCDF in the high column density end. We also notice the fact that the near- and mid-infrared extinction method is limited to detecting a lower range of column density $\left(A_{v} \sim 100\right.$, according to Kainulainen \& Tan 2013). Therefore, to evaluate the origin of this difference in the high column density regime, one should perform systematic error estimates of the extinction method, which is beyond the scope of our current analysis.

The sharp decrease for G28.34+0.06 and G14.225-0.506 at less than $\mathrm{N}\left(\mathrm{H}_{2}\right) \sim 4.0 \times 10^{22} \mathrm{~cm}^{-2}$ and the near flat region up to $\mathrm{N}\left(\mathrm{H}_{2}\right)<\sim 8.0 \times 10^{22} \mathrm{~cm}^{-2}$ may place these clouds at an evolutionary stage in between G11.11-0.12 and OB-clusterforming samples. These results imply that these two IRDCs already have a significant concentration in mass at relatively high column densities.

\section{Discussion}

\subsection{Indications of Physical Mechanisms Based on N-PDF and 2PT Results}

In this section, we compare the three observed IRDCs in terms of their N-PDFs results, and how these are linked to possible physical processes at work. We also compare their measured properties with observations of seven more evolved OB-cluster-forming regions reported in Lin et al. (2016). The masses and luminosities of all the sources are summarized in Figure 7. We caution that small sample size limits our analysis to being conjecturing rather than conclusive.

The N-PDFs of G11.11-0.12 and G14.225-0.506 have a more prominent log-normal component than OB-cluster-forming 

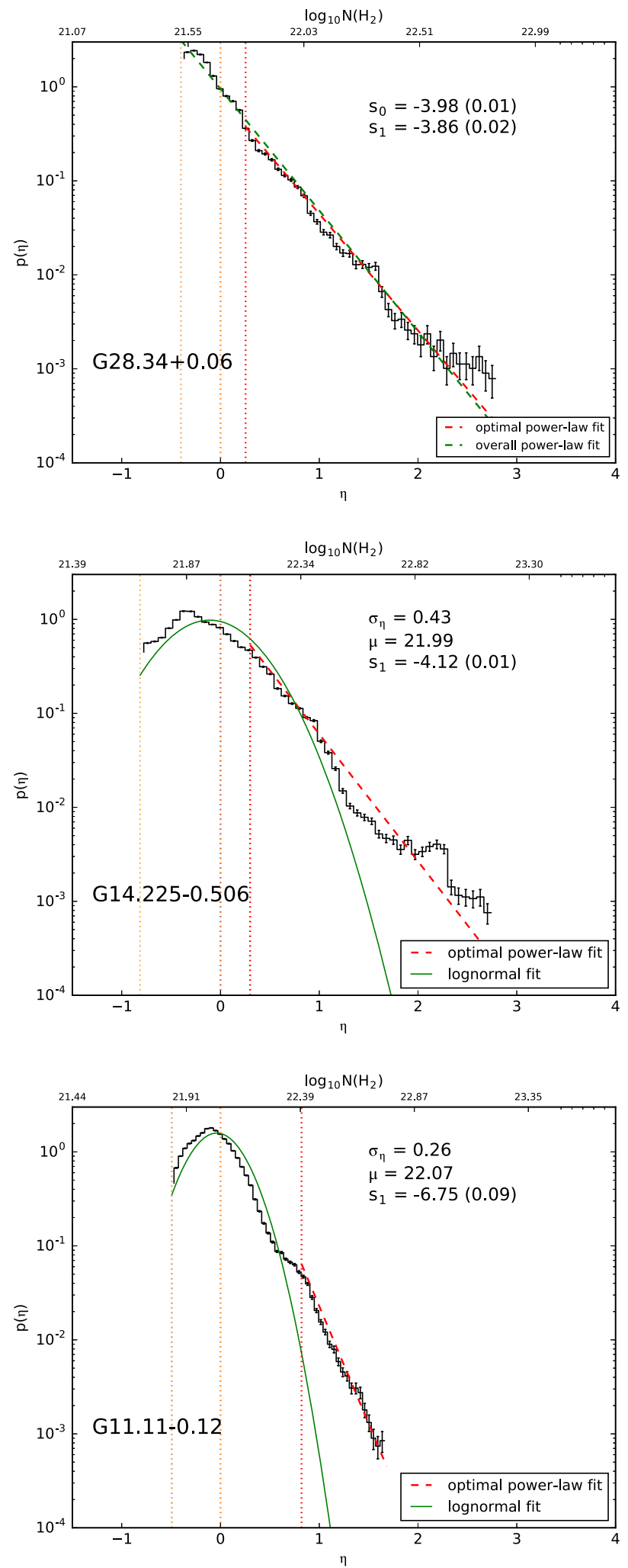

Figure 4. Column density probability distribution functions of the observed infrared dark clouds with light orange, orange, and red vertical dotted lines indicating the measured threshold of column density, mean column density, and column density starting value of the optimal power-law fit, respectively.

regions. Despite the possible bias induced by non-uniform sensitivity and different distances toward different sources, the N-PDF results in this paper and in Lin et al. (2016) are derived only for values greater than the measured column density

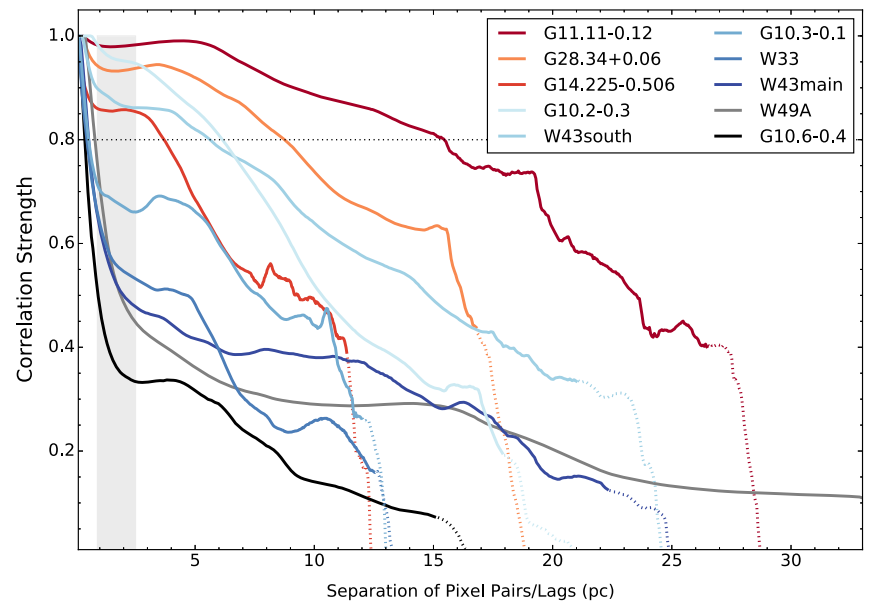

Figure 5. Two-point correlation functions of the column density distributions, for the observed sample. For separations with pixel pairs of less than an $\sim 90 \%$ total pixel number, the correlation strengths are plotted with dashed lines. The gray filled region indicates approximately where the rapidly decreasing components end for most of the OB-cluster-forming regions. The black horizontal dotted line indicates a correlation strength of 0.8 .

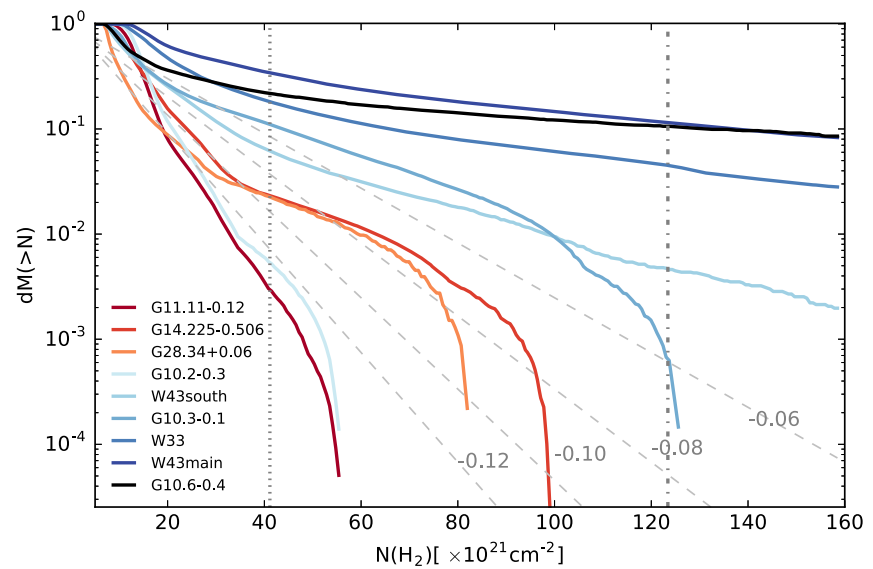

Figure 6. N-CCDF of three IRDCs and OB-cluster-forming regions. The lower threshold for all sources is $7 \times 10^{21} \mathrm{~cm}^{-2}$. All of the sources are smoothed to the resolution if they are at the distance of W43 $(\sim 5.5 \mathrm{kpc})$. The vertical dotted line indicates a core of $10^{5} \mathrm{~cm}^{-3}, \sim 0.1 \mathrm{pc}$, while the dashed dotted line indicates a core of $10^{6} \mathrm{~cm}^{-3}, \sim 0.03 \mathrm{pc}$ located at same distance. Color coding for each source is the same as in Figure 5.

threshold of each region and normalized to enable reliability in the comparisons. The log-normal plus power-law tail N-PDFs of G14.225-0.506 and G11.11-0.12 and their highly filamentary structures resemble, but are not uniquely explained by, the selfgravitating cylinder model (Myers 2015). The relative significance of the high-density part in the N-PDF of G14.225-0.506 is higher than in G11.11-0.12, with a larger $\sigma_{\eta}$ and a shallower power-law tail index $\alpha$. The increased spatial density concentration is also seen in the sources' 2PT functions: G14.225-0.506 has a more prominent decrease of correlation strength at small spatial scales. Turbulent flows or shocks in the large-scale, low column density regime may shape the initial morphology of these clouds, producing the log-normal component in the N-PDFs (Vazquez-Semadeni 1994; Klessen 2000). For example, Tackenberg et al. (2014) suggested that a large-scale accretion flow exists along the integral filament of G11.11-0.12. Unlike the extreme cases of W49A and G10.6-0.4, which may be undergoing global and local collapse simultaneously in the 


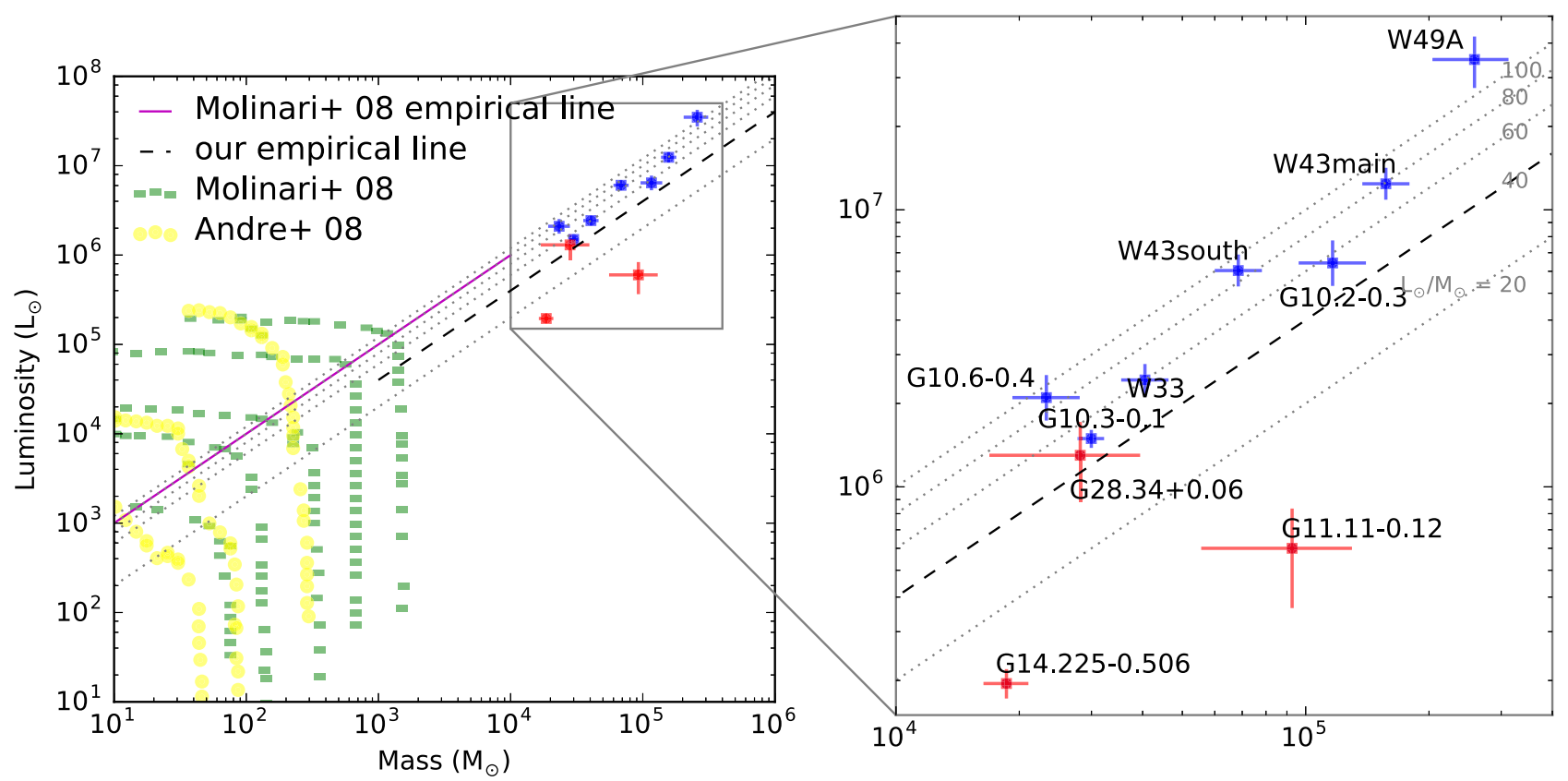

Figure 7. Luminosity-mass plot of all the sources. Error bars are calculated based on uncertainties of distances. For the kinematic distances of G11.11-0.12 and G28.34+0.06, we adopt a distance uncertainty of $20 \%$. The dashed black line denotes our empirical $L_{\odot} / M_{\odot}=40$ line. Dotted reference lines are $L_{\odot} / M_{\odot}=20,40,60,80,100$, respectively. Evolutionary tracks for final (formed) different masses of massive stars are indicated in yellow dashed lines (André et al. 2008) and for different initial envelope masses are indicated in green dashed lines (Molinari et al. 2008). The empirical boundary line of "Class 0"-- and "Class II"-like $\sim 1$ pc clumps derived by Molinari et al. (2008) is also indicated in the plot in magenta. Note that luminosities for OB-cluster-forming regions are different from those tabulated in Lin et al. (2016) where they were calculated by summing up all pixels in the fields of view.

clouds, the development of power-law tails at the high column density ends of G11.11-0.12 and G14.225-0.506 may suggest that they are only gravitationally unstable over a small fraction of the observed area.

We note that the N-PDFs of these two IRDCs may not necessarily be simply understood as being caused fully by the interplay between turbulence and self-gravity, considering that they both have been suggested to have dynamically nonnegligible magnetic fields (Pillai et al. 2015; Santos et al. 2016). The index of the N-PDF power-law tail and variance could be influenced by the magnetic field, as suggested by gravoturbulence simulations (Burkhart et al. 2015). In some of these simulations, the narrower standard deviation $\sigma$ of the log-normal part of the N-PDF is found to be caused by magnetic fields acting as a cushion that sets column densities closer to their average value (Nakamura \& Li 2008; Molina et al. 2012). The smaller variance and steep power-law tail of G11.11-0.12 seems to be compatible with these simulations. It has been suggested that the low column density end of the lognormal component may be biased due to limited image size (e.g., Lombardi et al. 2015). Our measurements might be affected by the completeness issue, hence, we avoid further discussions of the log-normal component in the present research.

The relation between changes in the N-PDF power-law tails and the evolutionary stage of molecular clouds is suggested by several recent observational and numerical simulation works. For example, Stutz \& Kainulainen (2015) investigate the variations in the N-PDF slopes with young star-formation content in the Orion molecular cloud, and they find that the $\mathrm{N}-\mathrm{PDF}$ slope is steeper in regions where there is a smaller fraction of Class 0 protostars. As the power-law tail flattens, the fraction of young protostars increases. The shallower powerlaw tail related to more active star formation is also suggested by Lombardi et al. (2015) based on investigations toward several molecular clouds. The possible relation between the power-law tail slopes and collapsing state of molecular clouds is also raised by Kritsuk et al. (2011), Ballesteros-Paredes et al. (2011), Burkhart et al. (2015) etc. in their simulations. Regarding cloud properties reflected by N-CCDF measures, Kainulainen et al. (2013a) find that the slope of N-CCDF relies sensitively on the turbulence driving mechanism and SFE, and magnetized simulations of clouds have steeper slopes than nonmagnetized ones. The 2PT functions of the selected IRDCs show slowly decaying correlation strengths at small spatial scales, indicating less matter concentration in local areas compared with most of the more luminous OB-cluster-forming regions. On the other hand, the IRDCs exhibit a larger decrease of correlation strengths at larger scales. The different (mixed) driving modes and magnetized nature of the turbulent motions in these IRDCs, compared to those in luminous OB-clusterforming regions, may be the origin of the above mentioned differences in our statistical measurements (Federrath et al. 2009).

The N-PDFs of G11.11-0.12 and G28.34+0.06 were also derived by Schneider et al. (2015) based on Herschel data only. Despite their different angular resolution, the overall shape of N-PDFs are similar, while we are able to resolve more localized dense structures. We note that the main caveat of utilizing N-PDF features to quantify cloud properties is that it is an indirect indicator of cloud physical state, unlike, i.e., volume density profiles. It is possible to convert column density maps to densities using simple assumptions of cloud geometry (Stutz \& Gould 2016), but this is not readily applicable to our sources considering their complex morphological structures based on previous spectroscopic works, which show that they have multiple velocity components. 


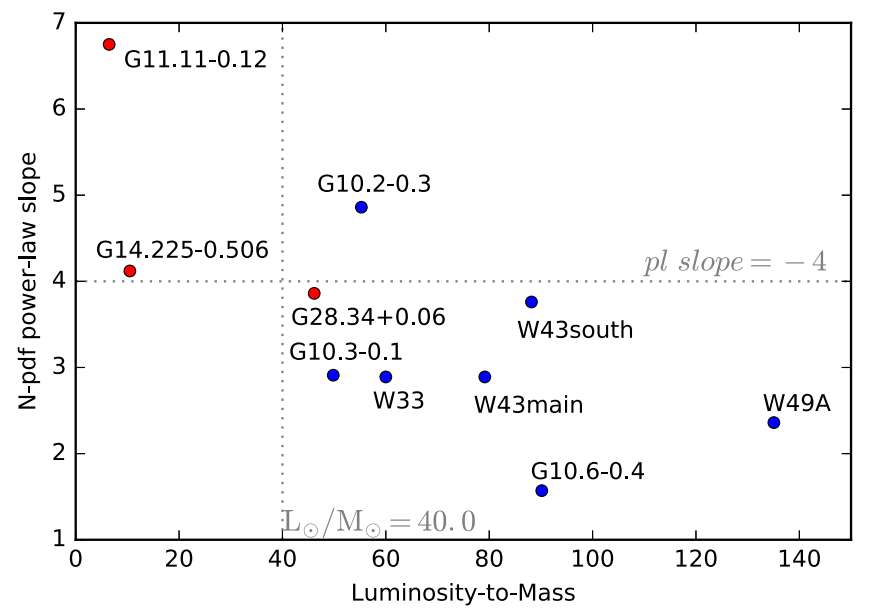

Figure 8. Luminosity-to-mass ratio vs. slope of N-PDF power-law at high column density for all of the sources. Luminosities and masses are in solar units.

In Figure 7, we plot the total luminosity versus mass of these sources with reference lines corresponding to constant luminosity-to-mass ratios. The luminosity-to-mass ratio provides a measure of evolutionary state. The ratio places G28.34 +0.06 in a similar stage with some of the OB-cluster-forming regions, whereas G14.225-0.506 and G11.11-0.12 are more quiescent regions. The slope of the N-PDF and the luminosityto-mass ratio of the compared sources are presented in Figure 8. The data points in the plot suggest a correlation between these two quantities.

\subsection{A Comparison with Simulations of Luminous OB-Cluster-formation}

We here compare our observations with recent numerical simulations by Dale et al. (2015), who followed the formation of OB clusters from molecular clouds, and examined the impact of the clusters' feedback on the clouds. The simulations were performed in the Smoothed Particle Hydrodynamics formalism. Smooth spherical clouds of a range of masses were initialized with a turbulent velocity field and allowed to evolve and form stars. Once a few massive stars had formed in each cloud, the effects of their winds and ionizing radiation were modeled. The evolution of the clouds followed as close as was practicable to the epoch when the massive stars were expected to explode as supernovae, allowing the influence of the winds and radiation to be isolated.

We choose the $10^{4} M_{\odot}$ Run I calculation from Dale et al, since it is among those with the best linear resolution. We compute simple column-density images along the simulation $z$-axis of $10 \times 10 \mathrm{pc}$ subfields of the cloud, comparable to the physical sizes of our observed IRDCs (see also Appendix B). Column-density images are produced at four epochs prior to the formation of stars and the initiation of feedback (top row of Figure 9), at the point when feedback begins to act (first panel in the bottom row of Figure 9), and for three later epochs (remaining panels in the bottom row of Figure 9).

The four images of the top row in Figure 9 show the development of self-gravitating filamentary structures from the cloud's turbulent velocity field before the onset of star formation. The gaseous filaments are initially not gravitationally unstable and, at early stages, their main role is to feed gas to a forming, parsec-scale massive molecular clump (the bright compact object located in the lower left corner of the two rightmost images in the top row). This quickly accumulates a large amount of mass and becomes the earliest site of star formation. In Figure 10, we compute N-PDFs for these column-density images in the same way as we did for the observational data. The absence of many massive molecular clumps in the simulated cloud is due to the initial dominance of global collapse.

At the earliest epoch when the filamentary structure is least well-defined, the PDF has a form close to log-normal in appearance. We caution that the drop-off at low $\eta$ is likely an incompleteness effect caused by the limited FOV and the lowest density contours not being closed. As the simulation progresses and the filaments become self-gravitating in preparation for forming stars, the N-PDFs develop pronounced power-law tails, which extend to higher and higher values of $\eta$, as do the observed plots (Figure 10). The slopes of the power laws are initially very steep, but flatten with time, approaching the slopes in the observed N-PDFs.

In even more striking resemblance to the observations of G14.225-0.506 (Figure 4), the simulated N-PDFs also shows a similar bump in the simulated data at $\eta \approx 2-2.5$ ((b)-(d) in Figure 9). This is dominated by the protocluster core mentioned earlier, indicating that material piles up in this structure for some time, slowing its progression to still higher densities and resulting in a bump in the N-PDF. There is also a minor contribution to the bump from the ambient dense gas filaments, which are immediately feeding the massive molecular clump. The massive molecular clump rapidly contracts due to selfgravity, which produces internal structures with high density and high column density. It then no longer appears as a bump in the N-PDF, whose slope extends further to higher values ((e) and (f) in Figure 9). The N-PDF evolves to a broken power-law shape at larger than zero $\eta$, showing an excess of high column density pixels. Such an N-PDF is similar to that observed in $\mathrm{G} 28.34+0.06$, and those of some active OB-cluster-forming regions (e.g., G10.6-0.4, W49A) reported in Lin et al. (2016). We note that an intermediate density bump present at volume density probability function $(\rho-\mathrm{PDF})$ in the cloud's early evolved phase is also seen in the formation of protoclusters simulated by Lee \& Hennebelle (2016).

In our simulations, the log-normal like N-PDF is thus not smoothly lifted to become a power-law like N-PDF, but instead changes via a rather dynamical process that produces nonsmooth and non-steady features. The bottom panels (e)-(h) of Figure 9 show the reaction of the N-PDF to feedback from the OB cluster, which eventually forms in the dense core. The massive molecular clump forms a condensed cluster of stars; some stars are ejected due to many-body gravitational interactions. Subsequently, the massive clump is destroyed by feedback and by consumption due to stellar accretion. Feedback of the stars formed in the massive clump then quickly disperse the low density gas and destroys the filamentary structure from which the clump originally formed. As a result, a cavity is gradually created at the center of the system. The action of feedback is initially rather modest and is essentially to drive the high-density tail to higher and higher values of $\eta$. The N-PDF at this stage shows a deficit of high column density gas when compared with a single power-law fit ( $(\mathrm{g})$ in Figure 9), which is qualitatively similar to the observed N-PDF of the evolved OB-cluster-forming region G10.2-0.3 (Lin et al. 2016). However, G10.2-0.3 presents several $\mathrm{H}$ II bubbles, which may 

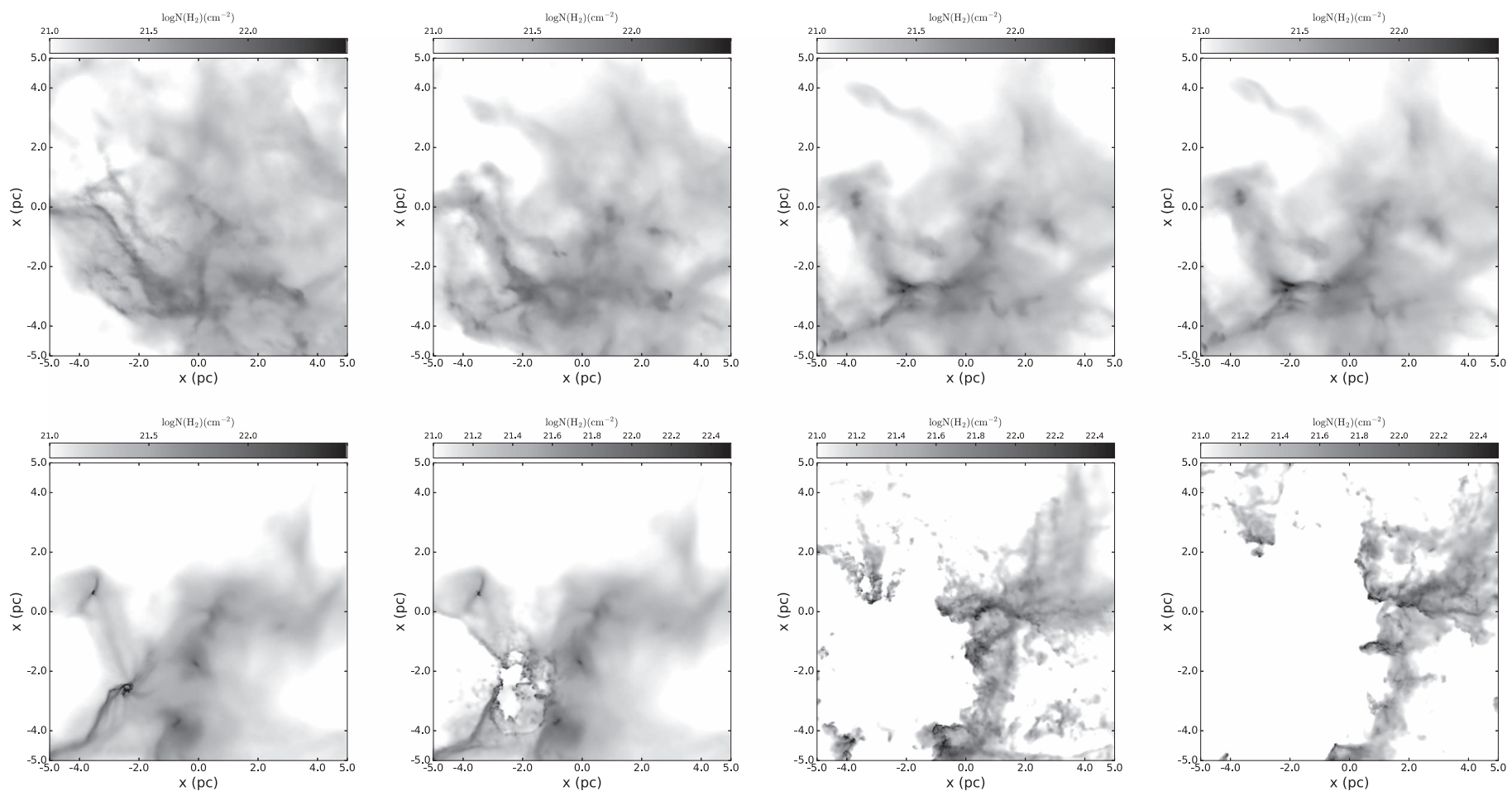

Figure 9. Simulated column density evolution of a $10^{4} M_{\odot}$ molecular cloud. Panels in the top row (from left to right), which are before the onset of star formation, show the time epoch of $2.4 \mathrm{Myr}, 3.2 \mathrm{Myr}, 3.7 \mathrm{Myr}$, and 3.9 Myr, respectively. Panels in the bottom (from left to right) show the time epoch of 5.4 Myr, $5.5 \mathrm{Myr}$, $6.0 \mathrm{Myr}$, and $6.5 \mathrm{Myr}$, respectively. Color scale of the first five epochs is the similar as that of our IRDC column density maps. More descriptions are in Section 4.2. The N-PDFs are summarized in Figure 10.
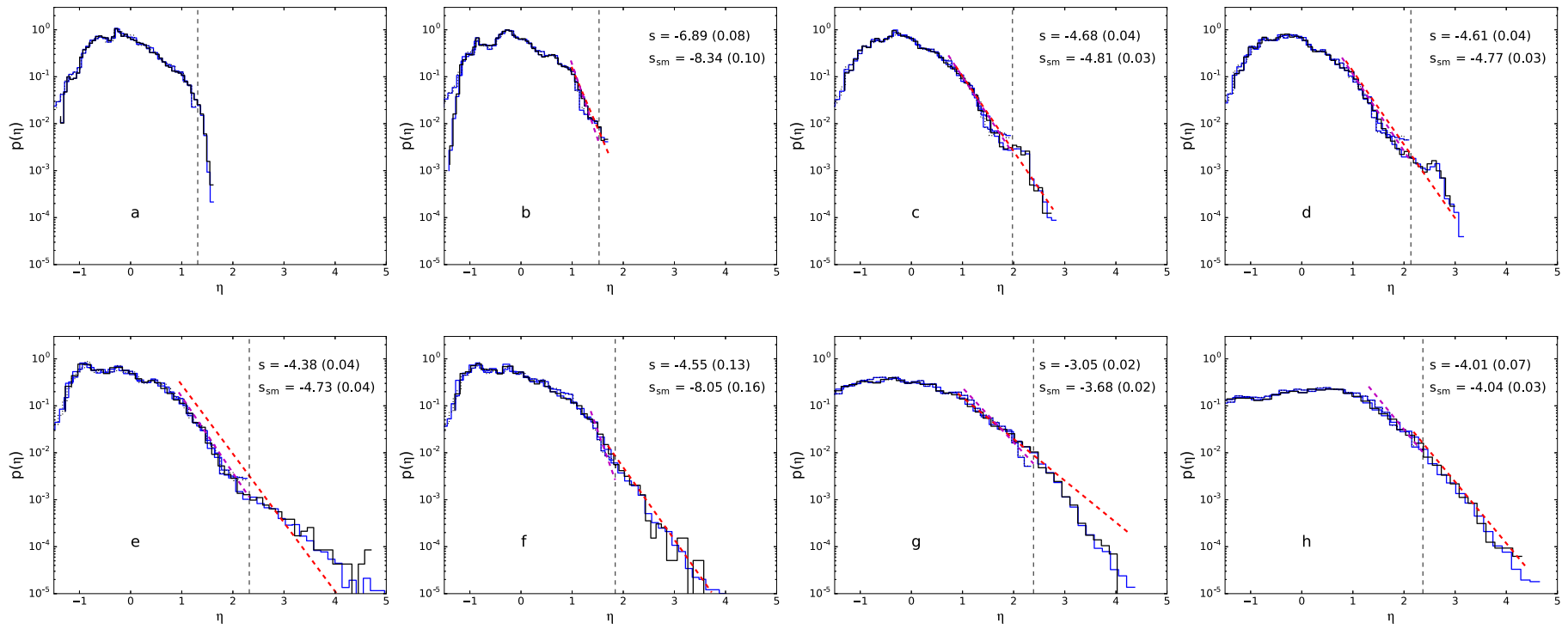

Figure 10. Simulated N-PDF evolution of a $10^{4} M_{\odot}$ molecular cloud. The gray histogram shows the simulated $4 \times 10^{6}$ data points, and the blue histogram shows the random sampled $2 \times 10^{5}$ data points (comparable to our observations) to fit with power-law distributions. The N-PDFs results after smoothing are shown by dotted histograms and largely overlap with the unsmoothed results with vertical dashed lines showing the truncations. Power-law slopes for two results are indicated in the graphs, where $s$ stands for the unsmoothed result and $s_{\mathrm{sm}}$ stands for the smoothed results.

shape the N-PDF differently. Eventually, the power-law slope of high column density gas becomes steeper again ((h) in Figure 9), as feedback becomes intense enough to significantly shape the high column density end. The smoothed N-PDFs shown in Figure 10 are simulated images smoothed to the resolution of our achieved resolution $10^{\prime \prime}$ at a median distance of our sources of $3 \mathrm{kpc}$. The smoothed N-PDFs and their fitted optimal power-law tail slopes are close to the original ones except that they exhibit clear truncations, which means that smoothing and differing distances do not affect our conclusions significantly but that high-resolution column density maps are essential to robustly recover the suppressed power-law tails at the high column density end.

As discussed in detail in Dale et al, feedback in this cloud is able to generate dense gas by compressing the outer regions of the cloud into shells, but much of this gas fails to become 

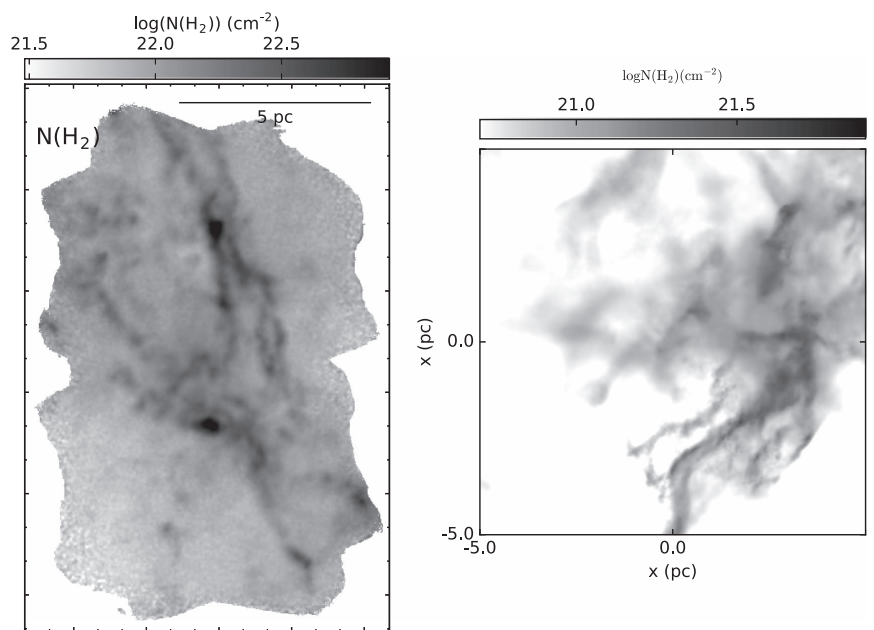

Figure 11. Left panel: G14.225-0.506 column density map and simulated image of time epoch 3.9 Myr. Right panel: G28.34+0.06 column density map and simulated image of time epoch 2.4 Myr. Spatial scale of simulated images is adjusted to be the same with corresponding sources.

gravitationally unstable because it is located far from the cloud's main potential well, and is stabilized by geometrical stretching and turbulence. We also examined one of the larger clouds modeled by Dale et al. (2015), the $3 \times 10^{5} M_{\odot}$ UUCL cloud). We obtained qualitatively similar results to those obtained for Run I, though the coarser mass and length resolution of Run UUCL does not allow the high-density end of the PDFs to be resolved as is possible in Run I.

\subsection{Characterizing the Cloud Evolutionary Stage Based on Cloud Structures and Temperature/Luminosity Profiles}

In the field of high-mass star formation, the evolutionary stages of massive (e.g., $M_{\text {gas }}>10^{4} M_{\odot}$ ) molecular clouds are commonly separated into the infrared dark stage, the active star-forming stage, and the dispersed stage. IRDCs are considered to be the youngest evolutionary stage, representing the initial conditions of high-mass star formation. However, unlike low-mass stars, high-mass stars form in much shorter (Kelvin-Helmholtz cooling) timescales than the characteristic (free-fall) timescales for molecular cloud evolution. Therefore, characterizing molecular cloud evolution based on the illumination from embedded high-mass stars, may be coarse and biased. In terms of terminology, whether a molecular cloud is infrared dark or not may also depend on the sensitivity and the FOV of the observations, and the time variability of the observed stellar emission, which can be ambiguous (e.g., Feng et al. 2016).

Establishing evolutionary tracks of massive molecular clouds based on their structures may be more essential and crucial for facilitating studies of the co-evolution of the (proto) stellar and core mass functions with the molecular cloud (e.g., Zhang et al. 2015). Based on a limited sample (the present work and Lin et al. 2016), and also based on the results of hydrodynamic simulations (Section 4.2), we hypothesize four common characteristic evolutionary stages of massive molecular clouds. From the earlier to the later evolutionary stages, they are (1) the cloud integration stage, (2) the stellar assembly stage, (3) the cloud pre-dispersal stage, and (4) the dispersed cloud stage.

In the cloud integration stage, high column density (e.g., $\mathrm{N}\left(\mathrm{H}_{2}\right)$ $\geqslant 10^{22} \mathrm{~cm}^{-2}$ ) gas structures are forming on $>10 \mathrm{pc}$ scales. At this stage, the N-CCDF remains steep. The gas/dust temperature may be $<20 \mathrm{~K}$ and the gas temperature is, in general, anti-correlated with the gas/dust column density, despite a few hot spots probably associated with deeply embedded intermediate-/highmass (proto)stars. Cloud structures at this stage are characterized by a steep and decreasing slope of the power-law tail in the N-PDFs, and the diffuse nature of the 2PTs do not show a significantly distinguishable component in the short lags. In $\sim 10$ pc scales, the low column density part of the N-PDFs may be described by log-normal distributions, until the self-gravitational contraction outweigh other physical mechanisms present from the initial conditions (e.g., turbulence, magnetic field, etc.). In addition, the N-CCDF and the luminosity-to-mass ratios are both low.

In the stellar assembly stage, the slopes of the power-law tail in the N-PDFs become shallower due to the overall selfgravitational contraction of the molecular cloud. In addition, the highest column density end of the N-PDF may start to present an excess of single power-law tail due to the presence of very massive molecular clumps/cores, which are forming the highest mass stars of the final clusters. The 2PT functions at this stage start to present a distinguishable component at lags $\lesssim 1 \mathrm{pc}$, which is related to the presence of the above mentioned very massive molecular clumps/cores. The luminosity-to-mass ratio at this stage increases very rapidly due to the quickly forming high-mass stars. From the two-dimensional histogram of the gas/dust temperature and column density, we can also identify positive correlations from the core/clump material of the immediate surroundings of the newly formed high-mass stars.

In the cloud pre-dispersal stage, the remaining dense gas associated with massive molecular clumps/cores is either accreted onto the young massive star(s) (Ginsburg et al. 2016), or dispersed by the stellar feedback (Dale et al. 2015). The distinguishable component at the $\lesssim 1 \mathrm{pc}$ lags of the 2PT functions become less prominent or it fully disappears. The slope of the power-law tail in the N-PDFs remains shallower than that in the cloud integration stage. The luminosity-to-mass ratio and the dense gas fraction at this stage will remain high as well. It is until the dispersed cloud stage that radiative feedback and the expansion of the ionized gas become capable of reverting the contracting motions on all spatial scales, significantly re-shaping molecular gas structures. The N-PDF becomes steeper back again and may show an increasing slope toward the high column density end. This evolutionary stage can be easily distinguished from the cloud integration stage and other evolutionary stages with similar N-PDF slopes because the gas/dust temperatures are much higher, and the presence of extended mid-infrared and radio continuum emission, which trace the extended and expanding $\mathrm{H}$ II regions. Whether the $\mathrm{N}-\mathrm{PDF}$ can be reverted by the feedback or not, may also depend on the initial cloud morphology (e.g., the cross-section against feedback). In the case in which the stellar feedback is only strong enough to disperse low density gas, the piled up high-density gas may re-collapse in some free-fall timescales, recycling again.

We tentatively suggest that the cloud integration stage and the dispersed cloud stage can be separated from the stellar assembly stage and cloud pre-dispersal stage by the $s_{1}=-4$ power-law index of the N-PDF, and by the correlation strength at the short-lag break in the 2PT function of correlation strength 0.8 . The cloud integration stage may be further separated from 
the three more evolved stages by a luminosity-to-mass ratio $\sim 40 L_{\odot} / M_{\odot}$. The stellar assembly stage may have a more prominent decrease of $2 \mathrm{PT}$ at small scales than the cloud pre-dispersal stage, with the latter possibly exhibiting a deficit of the singular power-law tail of N-PDF at the high column density end. We refer to Molinari et al. (2016) for an observed relation between the luminosity-to-mass ratio and the gas temperature of high-mass molecular clumps. The cloud predispersal stage and the cloud dispersed stage may be separated by $s_{1} \sim-4$ as well. Exactly how the cloud pre-dispersal stage and the cloud dispersed stage are separated from the N-PDF may be sensitive to the cloud initial condition and the subsequent star-formation activities. The more precise loci in the parameter space of the power-law slope of N-PDF, the 2PT function, the N-CCDF, and the luminosity-to-mass ratios, need to be defined with a larger survey. In this sense, the molecular cloud structures of G14.225-0.506 and G28.34+0.06 are on the transition between the cloud integration and the stellar assembly stages, when the massive molecular clumps/cores are forming. The massive clump P2 in G28.34+0.06 already formed high-mass stars, which is a quicker process than the evolution of cloud structures. The wiggling nature of the N-PDF of G14.225-0.506 with a "bump" in between $\eta=2-3$ may be due to the fact that very massive, parsec-scale forming molecular clumps have not yet reached their highest column densities (see also discussion in Section 4.2). W43-south has evolved to a stage where its luminosity-to-mass ratio is higher than W43-main, while its N-PDF is steeper, the N-CCDF is lower, and has less significant embedded massive molecular clump(s), as compared with W43-Main. The OB-clusterforming region G10.2-0.3 appears to be already dispersed by feedback mechanisms, such that its N-PDF has a steep slope, and N-CCDF is as low as the very young source G11.11 -0.12 . We note that molecular clouds may have different morphological classes (Lin et al. 2016). The parameters that separate the evolutionary stages may vary with the morphology classes, which needs to be examined with larger samples. However, the present difficulty in assembling a large sample is originated from the distance uncertainty/ambiguity. Based on the VLA survey of $\mathrm{NH}_{3}$ lines toward 62 high-mass starforming regions with $L_{\text {bol }} \sim 10^{4} L_{\odot}$, Lu et al. (2014) proposed that the clouds can be separated into the different morphological classes of filaments, concentration, and dispersed and a sub-sample not classified yet. These morphological classes may also be related to the evolutionary stages here proposed, if not purely determined by initial conditions.

\section{Conclusion}

We optimized the method of image combination described in Liu et al. (2015) and Lin et al. (2016) to extend our cloud structure analysis to three IRDCs. We examined their column density distributions via N-PDF, 2PT, and N-CCDF functions, and compared to those of OB-cluster-forming regions. The main findings are as follows.

1. The column density probability distribution functions (NPDFs) of G11.11-0.12 and G14.225-0.506 have steep power-law tails at their high column density end, while N-PDF of G28.34+0.06 exhibits a single power-law distribution. Comparing to the N-PDFs of OB-clusterforming regions, the power-law tails of these IRDCs are generally steeper. The shallowing PDFs with evolutionary state indicate the dominant role of gravitational collapse on the scales we probe. Evidence of power-law tail becoming steeper again at late stage when feedback is significant is also revealed by our simulations.

2. The two-point correlation functions (2PTs) of the three IRDCs generally decrease slowly over the observed spatial scales, in stark contrast to most of the OB-clusterforming clouds in our sample, which have a steep decay of correlation strength at small spatial scales. This indicates that IRDCs are much less concentrated than OB-cluster-forming clouds, since their column density distributions are more homogeneous at scales $>1 \mathrm{pc}$.

3 . The column density complementary cumulative distribution function (N-CCDF) of G11.11-0.12 and G28.34 +0.06 show a rapid decrease in their high column density range, similar to the most evolved OB-cluster-forming region $\mathrm{G} 10.2-0.3$. On the other hand, G14.225-0.506 has an N-CCDF behavior closer to the other OB-clusterforming regions.

4. Based on the observed correlations of N-PDF, 2PT, and $\mathrm{N}-\mathrm{CCDF}$ measurements with luminosity-to-mass ratios, we attempt to quantitatively characterize the evolution of high-mass star-forming molecular clouds based on the evolution of their (column) density distribution functions and spatial scales of gas structures. We hypothesize four common evolutionary stages, in spite of the possibility of different initial cloud morphology and supporting mechanisms (e.g., $B$-field, turbulence), namely, the cloud integration stage, the stellar assembly stage, the cloud pre-dispersal stage, and the dispersed cloud stage. The first and fourth stages exhibit similar patterns of N-PDF, 2PT, and N-CCDF, yet they can be distinguished from each other based on their rather different luminosity-tomass ratios. The second stage has a shallower N-PDF power law, prominent density distributions are constrained to smaller scales, as seen in their 2PT functions, and significant gas mass has accumulated in high-density regimes, as shown in their $\mathrm{N}-\mathrm{CCDF}$. The third stage has a slope of N-PDF power law still shallower than the first stage but its $2 \mathrm{PT}$ has a less prominent decreasing component at small scales and a possible deficit of power-law tail at the high column density end as compared to the second stage. Finally, the power-law tail of the N-PDF of the final stage becomes steeper than the third stage.

The comparisons made between IRDCs and OB-clusterforming regions with these column density related measurements are necessary in an evolutionary view, in addition to comparing their different morphologies. The physical mechanisms working at distinct evolutionary stages can be reflected by the parameters of these measurements. We note that future observations of a larger sample would be key to span parameter space and separate the different stages unambiguously.

We are grateful to our referee for helpful comments that led to improvement of the paper. This work is partially supported by the International Partnership Program of Chinese Academy of Sciences, grant No. 114A11KYSB20160008 and National Natural Science Foundation of China No. 11373038. Y.X.L. acknowledge Bo Zhang and Guangxing Li for useful suggestions and discussions. G.B. acknowledges the support of the Spanish Ministerio de Enconomia y Competitividad (MINECO) under 
grant FPDI-2013-18204. G.B. is supported by the Spanish MINECO grant AYA2014-57369-C3-1-P. Z.Y.Z. acknowledges support from the European Research Council in the form of the Advanced Investigator Programme, 321302, COSMICISM. R.G.-M. acknowledges support from UNAM-PAPIIT program IA102817. K.W. acknowledges support from Deutsche Forschungsgemeinschaft (DFG) grant WA3628-1/1 through priority program 1573 (Physics of the Interstellar Medium). The JCMT is operated by the East Asian Observatory on behalf of The National Astronomical Observatory of Japan, Academia Sinica Institute of Astronomy and Astrophysics, the Korea Astronomy and Space Science Institute, the National Astronomical Observatories of China and the Chinese Academy of Sciences (grant No. XDB09000000), with additional funding support from the Science and Technology Facilities Council of the United Kingdom and participating universities in the United Kingdom and Canada. The JCMT has historically been operated by the Joint Astronomy Centre on behalf of the Science and Technology Facilities Council of the United Kingdom, the National Research Council of Canada and the Netherlands Organization for Scientific Research. Additional funds for the construction of Q2 SCUBA-2 were provided by the Canada Foundation for Innovation.

\section{Appendix A \\ Brief Description of SED Fitting Procedure}

We briefly summarize our methods for deriving the column density and dust temperature maps here. We adopt a dust opacity $\kappa_{\nu}$ from Ossenkopf \& Henning (1994), where $\kappa_{230}=0.9 \mathrm{~cm}^{2} \mathrm{~g}^{-1}$ is the dust opacity for thin ice mantles with $10^{6} \mathrm{~cm}^{-2}$ gas density without coagulation (frequently referred to as $\mathrm{OH} 5$ ) at $230 \mathrm{GHz}$ and a gas to dust ratio $M_{g} / M_{d}$ of 100. As the modified blackbody assumption, the flux density $S_{\nu}$ at a certain observing frequency $\nu$ is given by

$$
S_{\nu}=\Omega_{m} B_{\nu}\left(T_{d}\right)\left(1-e^{-\tau_{\nu}}\right)
$$

and

$$
N_{\mathrm{H}_{2}}=\frac{\tau_{\nu} M_{g}}{\kappa_{\nu} \mu m_{\mathrm{H}} M_{d}},
$$

where $B_{\nu}\left(T_{d}\right)$ is the Planck function at a given temperature $T_{d}$, and

$$
\kappa_{\nu}=\kappa_{230}\left(\frac{\nu}{230 \mathrm{GHz}}\right)^{\beta} \text {, }
$$

where $\beta$ is the dust opacity index. We adopt a dust opacity law similar to Hildebrand (1983). In the iterative SED fits, we first use 70, $160 \mu \mathrm{m}$ from PACS and combined SPIRE $350 \mu \mathrm{m}$ with SHARC2 $350 \mu \mathrm{m}$, combined Herschel extrapolated $850 \mu \mathrm{m}$ with SCUBA2/LABOCA and PLANCK353 GHz data to simultaneously fit $\beta$, gas column density and dust temperature. In the final fits, we use $70 \mu \mathrm{m}$ and combined $350 \mu \mathrm{m}$ with derived $\beta$ from former fits to derive gas column densities and dust temperature to achieve the best angular resolution of $10^{\prime \prime}$. For the discussion of potential uncertainties, we refer to Section 2.4 and Appendix C in Lin et al. (2016).

\section{Appendix B \\ Morphological Comparison of IRDC G14.225-0.506 and G28.34+0.06 with Our Simulated Images}

Morphologically, our simulated images resemble with these IRDCs. We include these early epoch simulated images (direction flipped as compared to Figure 9) side-by-side with our derived column density maps of G14.225-0.506 and G28.34+0.06 in Figure 11. The geometrical resemblance we see here is suggests good support for our comparisons of $\mathrm{N}-\mathrm{PDF}$ between simulated images and our observations.

\section{References}

Alstott, J., Bullmore, E., \& Plenz, D. 2014, PLoS One, 9, e85777 André, P., Minier, V., Gallais, P., et al. 2008, A\&A, 490, L27 Ballesteros-Paredes, J., Vázquez-Semadeni, E., Gazol, A., et al. 2011, MNRAS, 416, 1436

Burkhart, B., Collins, D. C., \& Lazarian, A. 2015, ApJ, 808, 48 Busquet, G., Estalella, R., Palau, A., et al. 2016, ApJ, 819, 139 Busquet, G., Zhang, Q., Palau, A., et al. 2013, ApJL, 764, L26 Butler, M. J., Tan, J. C., \& Kainulainen, J. 2014, ApJL, 782, L30 Carey, S. J., Clark, F. O., Egan, M. P., et al. 1998, ApJ, 508, 721 Carey, S. J., Feldman, P. A., Redman, R. O., et al. 2000, ApJL, 543, L157 Chapin, E. L., Berry, D. S., Gibb, A. G., et al. 2013, MNRAS, 430, 2545 Chen, H.-R., Liu, S.-Y., Su, Y.-N., \& Wang, M.-Y. 2011, ApJ, 743, 196 Clauset, A., Rohilla Shalizi, C., \& Newman, M. E. J. 2009, SIAMR, 51,661

Dale, J. E., Ercolano, B., \& Bonnell, I. A. 2015, MNRAS, 451, 987

Dempsey, J. T., Friberg, P., Jenness, T., et al. 2013, MNRAS, 430, 2534

Federrath, C., Klessen, R. S., \& Schmidt, W. 2009, ApJ, 692, 364

Feng, S., Beuther, H., Zhang, Q., et al. 2016, ApJ, 828, 100

Gao, Y., \& Solomon, P. M. 2004, ApJ, 606, 271

Ginsburg, A., Bally, J., Battersby, C., et al. 2015, A\&A, 573, A106

Ginsburg, A., Goss, W. M., Goddi, C., et al. 2016, A\&A, 595, A27

Griffin, M. J., Abergel, A., Abreu, A., et al. 2010, A\&A, 518, L3

Hennebelle, P., Pérault, M., Teyssier, D., \& Ganesh, S. 2001, A\&A, 365, 598

Henning, T., Linz, H., Krause, O., et al. 2010, A\&A, 518, L95

Hildebrand, R. H. 1983, QJRAS, 24, 267

Holland, W. S., Bintley, D., Chapin, E. L., et al. 2013, MNRAS, 430, 2513

Inutsuka, S.-I., \& Miyama, S. M. 1997, ApJ, 480, 681

Kainulainen, J., Federrath, C., \& Henning, T. 2013a, A\&A, 553, L8

Kainulainen, J., Ragan, S. E., Henning, T., \& Stutz, A. 2013b, A\&A, 557, A120

Kainulainen, J., \& Tan, J. C. 2013, A\&A, 549, A53

Kauffmann, J., \& Pillai, T. 2010, ApJL, 723, L7

Kleiner, S. C., \& Dickman, R. L. 1984, ApJ, 286, 255

Klessen, R. S. 2000, ApJ, 535, 869

Kóvacs, A. 2008, Proc. SPIE, 7020, 70201S

Kritsuk, A. G., Norman, M. L., \& Wagner, R. 2011, ApJL, 727, L20

Lada, C. J., Lombardi, M., \& Alves, J. F. 2010, ApJ, 724, 687

Lee, Y.-N., \& Hennebelle, P. 2016, A\&A, 591, A30

Lin, Y., Liu, H. B., Li, D., et al. 2016, ApJ, 828, 32

Liu, H. B., Galván-Madrid, R., Jiménez-Serra, I., et al. 2015, ApJ, 804, 37

Liu, H. B., Jiménez-Serra, I., Ho, P. T. P., et al. 2012a, ApJ, 756, 10

Liu, H. B., Quintana-Lacaci, G., Wang, K., et al. 2012b, ApJ, 745, 61

Lombardi, M., Alves, J., \& Lada, C. J. 2015, A\&A, 576, L1

Lu, X., Zhang, Q., Liu, H. B., Wang, J., \& Gu, Q. 2014, ApJ, 790, 84

Molina, F. Z., Glover, S. C. O., Federrath, C., \& Klessen, R. S. 2012, MNRAS, 423, 2680

Molinari, S., Merello, M., Elia, D., et al. 2016, ApJL, 826, L8

Molinari, S., Pezzuto, S., Cesaroni, R., et al. 2008, A\&A, 481, 345

Molinari, S., Swinyard, B., Bally, J., et al. 2010, A\&A, 518, L100

Myers, P. C. 2015, ApJ, 806, 226

Nakamura, F., \& Li, Z.-Y. 2008, ApJ, 687, 354

Ossenkopf, V., \& Henning, T. 1994, A\&A, 291, 943

Peretto, N., \& Fuller, G. A. 2009, A\&A, 505, 405

Pillai, T., Kauffmann, J., Tan, J. C., et al. 2015, ApJ, 799, 74

Pillai, T., Wyrowski, F., Menten, K. M., \& Krügel, E. 2006, A\&A, 447, 929

Planck Collaboration, Abergel, A., Ade, P. A. R., et al. 2011, A\&A, 536 A25

Planck Collaboration, Ade, P. A. R., Aghanim, N., et al. 2014, A\&A, 564, A45 Planck HFI Core Team, Ade, P. A. R., Aghanim, N., et al. 2011a, A\&A, 536, A6 
Planck HFI Core Team, Ade, P. A. R., Aghanim, N., et al. 2011b, A\&A, 536, A4

Poglitsch, A., Waelkens, C., Geis, N., et al. 2010, A\&A, 518, L2

Ragan, S. E., Bergin, E. A., Plume, R., et al. 2006, ApJS, 166, 567

Rosero, V., Hofner, P., McCoy, M., et al. 2014, ApJ, 796, 130

Santos, F. P., Busquet, G., Franco, G. A. P., Girart, J. M., \& Zhang, Q. 2016, ApJ, 832, 186

Schneider, N., Csengeri, T., Klessen, R. S., et al. 2015, A\&A, 578, A29

Simon, R., Rathborne, J. M., Shah, R. Y., Jackson, J. M., \& Chambers, E. T. 2006, ApJ, 653, 1325

Siringo, G., Kreysa, E., Kovács, A., et al. 2009, A\&A, 497, 945

Sridharan, T. K., Beuther, H., Saito, M., Wyrowski, F., \& Schilke, P. 2005, ApJL, 634, L57

Stutz, A. M., \& Gould, A. 2016, A\&A, 590, A2

Stutz, A. M., \& Kainulainen, J. 2015, A\&A, 577, L6

Tackenberg, J., Beuther, H., Henning, T., et al. 2014, A\&A, 565, A101
Vazquez-Semadeni, E. 1994, ApJ, 423, 681

Vázquez-Semadeni, E., Gómez, G. C., Jappsen, A. K., et al. 2007, ApJ, 657, 870

Vázquez-Semadeni, E., Gómez, G. C., Jappsen, A.-K., Ballesteros-Paredes, J., \& Klessen, R. S. 2009, ApJ, 707, 1023

Wang, K. 2015, The Earliest Stages of Massive Clustered Star Formation: Fragmentation of Infrared Dark Clouds: Springer Theses (Berlin: Springer) Wang, K., Zhang, Q., Testi, L., et al. 2014, MNRAS, 439, 3275

Wang, K., Zhang, Q., Wu, Y., Li, H.-B., \& Zhang, H. 2012, ApJL, 745, L30

Wang, K., Zhang, Q., Wu, Y., \& Zhang, H. 2011, ApJ, 735, 64

Wang, Y., Zhang, Q., Pillai, T., Wyrowski, F., \& Wu, Y. 2008, ApJL, 672, L33

Wu, J., Evans, N. J., II, Gao, Y., et al. 2005, ApJL, 635, L173

Wu, Y. W., Sato, M., Reid, M. J., et al. 2014, A\&A, 566, A17

Xu, Y., Moscadelli, L., Reid, M. J., et al. 2011, ApJ, 733, 25

Zacchei, A., Maino, D., Baccigalupi, C., et al. 2011, A\&A, 536, A5

Zhang, Q., Wang, K., Lu, X., \& Jiménez-Serra, I. 2015, ApJ, 804, 141

Zhang, Q., Wang, Y., Pillai, T., \& Rathborne, J. 2009, ApJ, 696, 268 


\title{
Erratum: "Cloud Structure of Three Galactic Infrared Dark Star-forming Regions from Combining Ground and Space-based Bolometric Observations” (2017, ApJ, 840, 22)
}

\author{
Yuxin Lin ${ }^{1,2}$, Hauyu Baobab Liu ${ }^{3}$, James E. Dale ${ }^{4}$, Di Li ${ }^{1,5}$, Gemma Busquet ${ }^{6}$, Zhi-Yu Zhang ${ }^{3,7}$, Adam Ginsburg ${ }^{3}$, \\ Roberto Galván-Madrid $^{8}$, Attila Kovács ${ }^{9}$, Eric Koch ${ }^{10}$, Lei Qian ${ }^{1,5}$, Ke Wang ${ }^{3}$, Steve Longmore ${ }^{11}$, \\ Huei-Ru Chen ${ }^{12}$, and Daniel Walker ${ }^{11}$ \\ ${ }^{1}$ National Astronomical Observatories, Chinese Academy of Sciences, China \\ ${ }^{2}$ Max-Planck-Institut für Radioastronomie, D-53121 Bonn, Germany; ylin@mpifr-bonn.mpg.de \\ ${ }^{3}$ European Southern Observatory (ESO), Karl-Schwarzschild-Str. 2, D-85748 Garching, Germany \\ ${ }^{4}$ Centre for Astrophysics Research, University of Hertfordshire, College Lane, Hatfield, AL10 9AB, UK \\ ${ }_{5}$ Key Laboratory of Radio Astronomy, Chinese Academy of Sciences, China \\ ${ }^{6}$ Institut de Ciències de l'Espai (IEEC-CSIC), Campus UAB, Carrer de Can Magrans S/N, 08193, Barcelona, Catalunya, Spain \\ ${ }^{7}$ Institute for Astronomy, University of Edinburgh, Royal Observatory, Blackford Hill, Edinburgh EH9 3HJ, UK \\ ${ }^{8}$ Instituto de Radioastronomía y Astrofísica, UNAM, Apdo. Postal 3-72 (Xangari), 58089 Morelia, Michoacán, México \\ ${ }^{9}$ California Institute of Technology 301-17, 1200 E California Boulevard, Pasadena, CA 91125, USA \\ ${ }^{10}$ Department of Physics, University of Alberta, 4-181 CCIS, Edmonton, AB T6G 2E1, Canada \\ ${ }^{11}$ Astrophysics Research Institute, Liverpool John Moores University, 146 Brownlow Hill, Liverpool L3 5RF, UK \\ ${ }^{12}$ Institute of Astronomy and Department of Physics, National Tsing Hua University, Hsinchu, Taiwan \\ Received 2017 June 2; published 2017 July 14
}

\section{Introduction}

Due to a mistake in proofreading, there is a sub-figure of Figure 11 missing in the paper, which corresponds to the second caption therein. The right figure and caption is below.

We also note that the reference empirical line of Molinari et al. (2008) in our Figure 7 is not accurately plotted, the slope of which should be $\sim 1.13-1.27$. While we do not use it to discuss our results since it is derived in a generally lower range of mass and luminosity of small-scale star-forming regions, we refer to the original work for a detailed description of this reference line.
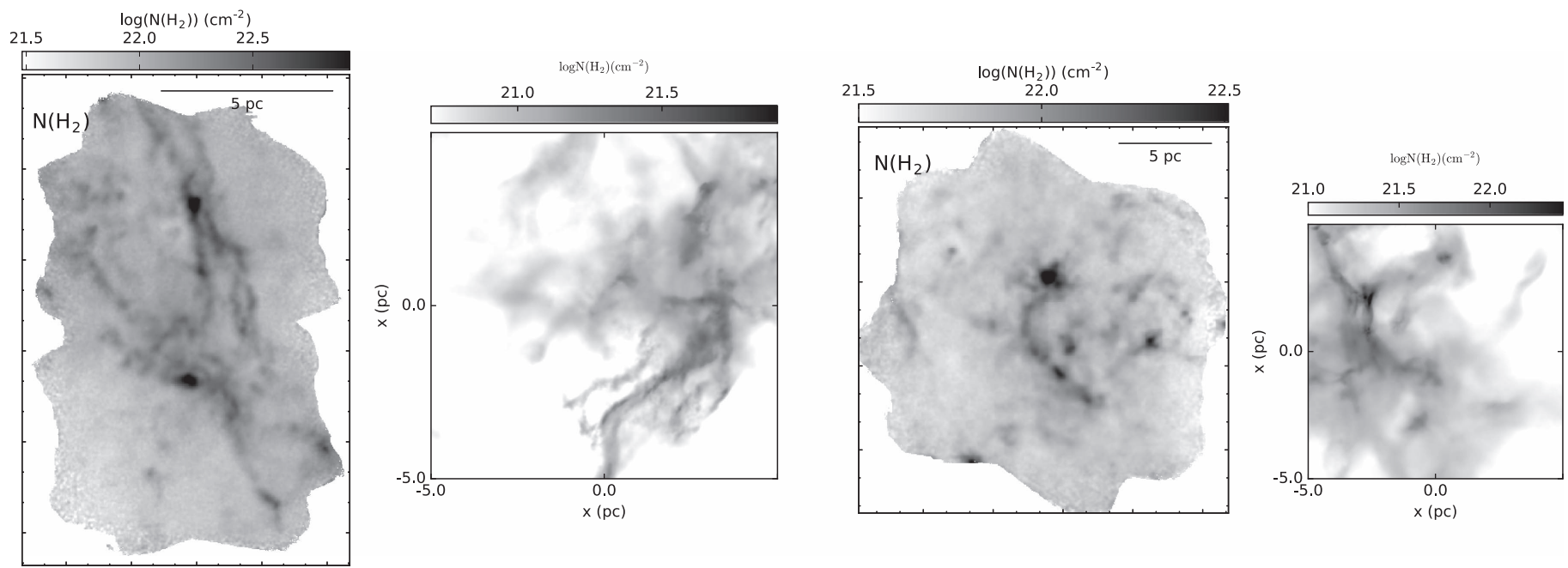

Figure 1. Left panel: G14.225 -0.506 column density map and simulated image of time epoch 3.9 Myr. Right panel: G28.34+0.06 column density map and simulated image of time epoch 2.4 Myr. In each panel, the spatial scale of the simulated image is adjusted to be the same as the corresponding source.

\section{Reference}

